\title{
ANALYSIS OF A STABILIZED FINITE ELEMENT APPROXIMATION FOR A LINEARIZED LOGARITHMIC REFORMULATION OF THE VISCOELASTIC FLOW PROBLEM
}

\author{
Ramon Codina $^{1,2, *}$ and Laura Moreno ${ }^{1}$
}

\begin{abstract}
In this paper we present the numerical analysis of a finite element method for a linearized viscoelastic flow problem. In particular, we analyze a linearization of the logarithmic reformulation of the problem, which in particular should be able to produce results for Weissenberg numbers higher than the standard one. In order to be able to use the same interpolation for all the unknowns (velocity, pressure and logarithm of the conformation tensor), we employ a stabilized finite element formulation based on the Variational Multi-Scale concept. The study of the linearized problem already serves to show why the logarithmic reformulation performs better than the standard one for high Weissenberg numbers; this is reflected in the stability and error estimates that we provide in this paper.
\end{abstract}

Mathematics Subject Classification. 65N12, 76A10, 76M10.

Received February 19, 2020. Accepted May 26, 2020.

\section{INTRODUCTION}

Computing viscoelastic fluid flows when the fluid presents a dominant elastic behavior is one of the biggest challenges of the computational rheology field nowadays. The equations that model the viscoelastic fluid flow problem present several instabilities that have been studied for example in [16,31]. These instabilities become important when elasticity becomes dominant, in other words, when the Weissenberg number is high. This dimensionless number is defined by $\mathrm{We}=\lambda u / L$, where $\lambda$ is the characteristic relaxation time, $u$ is the characteristic velocity of the flow and $L$ the characteristic length of the domain. Solving the viscoelastic fluid flow problem for high Weissenberg numbers is known as the High Weissenberg Number Problem (HWNP) [29], and it is usually described as a numerical phenomenon in which the iterative schemes breakdown even for moderate Weissenberg numbers. The numerical instability was studied by Fattal and Kupferman [14], who explained that it is present in the constitutive models and the standard numerical methods. Constitutive equations could present also other type of instabilities from a mathematical point of view, referred to as constitutive instabilities [24,25]. These can be classified in two: the Hadamard instability, which is a consequence of the non-linear fast response of the constitutive equation, and the dissipative instability, related to the dissipative behavior of viscoelastic models.

Keywords and phrases. Stabilized finite element methods, viscoelastic fluids, Oldroyd-B, logarithm reformulation, high Weissenberg number problem.

1 Universitat Politècnica de Catalunya, Jordi Girona 1-3, Edifici C1, Barcelona 08034, Spain.

2 CIMNE - Centre Internacional de Mètodes Numèrics en Enginyeria, Gran Capità S/N, Barcelona 08034, Spain.

*Corresponding author: ramon. codina@upc.edu 
However, the reasons for the HWNP are different: one is the loss of positive-definiteness of the conformation tensor, which is an internal variable that must be positive-definite to be physically admissible [14,21], and the second is the appearance of regions with large strain gradients.

From the numerical point of view, Fattal and Kupferman $[14,15]$ focussed the origin of the problem in the inappropriate approximations to represent the stress tensor, standing out the necessity of preserving the positivity of the conformation tensor. By following these ideas the logarithmic conformation reformulation was proposed in [14] as a formulation of the equations which overcomes the instability and linearizes the exponential stress profiles near the stress singularities. The formulation treats the exponential growth of the elastic stresses, allowing to extend the usual range of Weissenberg numbers which can be considered to simulate viscoelastic fluid flows. In this paper we will use a slightly different scaling of the logarithmic reformulation. In contrast with the original one, our change of variables will be non-singular when the Weissenberg number is close to zero and the flow is Newtonian [28]; a similar idea was presented in [33].

In addition to the instabilities mentioned, viscoelastic fluid flows present some compatibility restrictions when the Galerkin finite element (FE) approximation is undertaken. First, velocity and pressure FE spaces must satisfy the well known inf-sup condition for incompressible flows [4] and, secondly, there is another inf-sup condition that needs to be satisfied between stresses and velocities [32]. This is studied for example for the Navier-Stokes problem in [3,11]; the same requirements are met in the viscoelastic case. Stable interpolations are proposed in [27], whose analysis can be found in [17]. At the continuous level, these inf-sup conditions are satisfied and the problem can be shown to have a unique solution in the slow viscoelastic case [16,31]; see also $[18,19]$ for some results concerning strong solutions.

Referring to the FE approximation, there are several works that analyze the stationary Oldroyd-B problem and present optimal error estimates. For instance, Baranger and Sandri [1] is one of the first papers that shows the existence of $\mathrm{FE}$ approximations assuming that the continuous problem admits sufficiently smooth and sufficiently small solutions; bounds for the error are also provided. In [30], the authors establish the existence and a priori error estimates using the EVSS (Elastic Viscous Split Stress) method, aimed to circumvent the inf-sup condition between velocities and stresses. More recently, in [26] the authors present an error analysis of a particular Oldroyd-B model with the limiting Weissenberg number going to infinity, assuming a suitable regularity of the exact solution, for FE and finite volume methods. The time-dependent Oldroyd-B problem has also been studied, for example in [13], using the SUPG method, aimed to circumvent convection instabilities.

In this work, the log-conformation reformulation is applied together with a stabilized FE formulation based on the Variational Multi-Scale (VMS) method which aims at circumventing the inf-sup conditions and dealing with convection dominated flows at the same time. The VMS concept was presented first by Hughes et al. [20] for the convection-diffusion-reaction problem. These ideas were applied and extended in [8-11] for the NavierStokes problem and the three-field Stokes problem considering the space of the sub-grid scales orthogonal to the FE space. The viscoelastic fluid flow problem was stabilized following a VMS framework in $[6,7]$ and in the logarithmic formulation the method was tested in [28] for some numerical examples in which the Weissenberg number is relevant. This type of stabilization is also applied in [22,23].

The present paper can be considered a continuation of the work presented in [6,11]. In [11], a VMS formulation for the three-field Stokes problem was presented and analyzed. The same approach was followed in [6], in this case applied to a linearized version of the stationary standard formulation of the viscoelastic flow problem, using the Oldroyd-B model. The linearization is based on considering given the advection velocity and the velocity gradient in the rotational terms of the constitutive equation. The purpose of this paper is to extend this analysis to the logarithmic reformulation of the viscoelastic problem. This analysis will serve to explain its improved performance with respect to the standard formulation when the Weissenberg number is high. This would be difficult to observe in the full nonlinear problem, since conditions to ensure existence of solutions and their finite element approximation pose stringent requirements on the Reynolds and Weissenberg numbers of the problem, even in the time dependent case; for the standard formulation and using a similar approach as the one we follow here, this analysis can be found in [2]. 
This paper is organized as follows. In Section 2, the logarithmic formulation is described, starting from the standard viscoelastic Oldoryd-B fluid flow equations; Section 3 presents the FE method employed, based on the VMS approach. In Section 4, the numerical analysis is developed; in particular, in Section 4.2 stability and convergence are proved for a mesh dependent norm, while in Section 4.3 results are obtained for natural norms. Finally, conclusions are drawn in Section 5.

\section{THE MODIFIED LOG-CONFORMATION FORMULATION PROBLEM}

\subsection{Standard formulation for the viscoelastic flow problem}

Let us start presenting the standard equations associated to the viscoelastic flow problem. Let us consider a viscoelastic fluid moving in a domain $\Omega$ of $\mathbb{R}^{d}(d=2$ or 3 ) and let $\partial \Omega$ be the boundary. Assuming the flow to be incompressible and isothermal, the equations of the linearized problem we consider are:

$$
\begin{aligned}
& \rho \boldsymbol{a} \cdot \nabla \boldsymbol{u}-\nabla \cdot \boldsymbol{T}+\nabla p=\boldsymbol{f} \quad \text { in } \quad \Omega, \\
& \nabla \cdot \boldsymbol{u}=0 \quad \text { in } \Omega,
\end{aligned}
$$

where $\boldsymbol{a}$ is the advection velocity, $\rho$ denotes the constant density, $p: \Omega \rightarrow \mathbb{R}$ is the pressure field, $\boldsymbol{u}: \Omega \rightarrow \mathbb{R}^{d}$ is the velocity field and $\nabla \boldsymbol{u}$ its gradient, with Cartesian components $\left.\nabla \boldsymbol{u}\right|_{i j}=\partial_{i} u_{j}, i, j=1, \ldots, d, \partial_{i}$ being the derivative with respect to the $i$ th Cartesian coordinate, $f: \Omega \rightarrow \mathbb{R}^{d}$ is the force field and $\boldsymbol{T}: \Omega \rightarrow \mathbb{R}^{d} \otimes \mathbb{R}^{d}$ is the deviatoric extra stress tensor. In general, $\boldsymbol{T}$ is defined in terms of a viscous and a viscoelastic contribution as

$$
\boldsymbol{T}=2 \eta_{e} \nabla^{\mathrm{s}} \boldsymbol{u}+\boldsymbol{\sigma}
$$

where $\eta_{e}$ represents the effective viscosity (or solvent viscosity), $\nabla^{\mathrm{s}} \boldsymbol{u}$ is the symmetrical part of the velocity gradient and $\boldsymbol{\sigma}$ is the viscoelastic or simply elastic stress tensor.

Finally, the constitutive equation for the viscoelastic stress tensor must be defined to close the problem. Even if there is a wide range of different models, we consider the Oldroyd-B model in this work, which is the model of a Newtonian stress supplemented with an extra-stress that satisfies the upper-convected Maxwell equation. In the linearized version we consider it reads as

$$
\frac{1}{2 \eta_{p}} \boldsymbol{\sigma}-\nabla^{\mathrm{s}} \boldsymbol{u}+\frac{\lambda}{2 \eta_{p}}\left(\boldsymbol{a} \cdot \nabla \boldsymbol{\sigma}-\boldsymbol{\sigma} \cdot \nabla \boldsymbol{a}-(\nabla \boldsymbol{a})^{T} \cdot \boldsymbol{\sigma}\right)=\mathbf{0} \quad \text { in } \Omega,
$$

where $\lambda$ is the relaxation time and $\eta_{p}$ represents the polymeric viscosity. Each term of the equation has a particular meaning: $\nabla^{\mathrm{s}} \boldsymbol{u}$ is the source, $\frac{\lambda}{2 \eta_{p}} \boldsymbol{a} \cdot \nabla \boldsymbol{\sigma}$ represents the linearized convective term and $\frac{\lambda}{2 \eta_{p}}\left(\boldsymbol{\sigma} \cdot \nabla \boldsymbol{a}+\left(\nabla \boldsymbol{a}^{T}\right) \cdot \boldsymbol{\sigma}\right)$ are the linearized rotational terms. We write the polymeric and the effective viscosity in function of the total viscosity $\eta_{0}$; for that, an additional parameter $\beta \in[0,1]$ is introduced, so that $\eta_{e}=\beta \eta_{0}$ and $\eta_{p}=(1-\beta) \eta_{0}$.

Calling $\boldsymbol{U}=[\boldsymbol{u}, p, \boldsymbol{\sigma}], \boldsymbol{F}=[\boldsymbol{f}, 0, \mathbf{0}]$ and defining

$$
\mathcal{L}_{\mathrm{st}}(\boldsymbol{U}):=\left(\begin{array}{c}
-\nabla \cdot \boldsymbol{\sigma}-2 \eta_{e} \nabla \cdot\left(\nabla^{\mathrm{s}} \boldsymbol{u}\right)+\rho \boldsymbol{a} \cdot \nabla \boldsymbol{u}+\nabla p \\
\nabla \cdot \boldsymbol{u} \\
\frac{1}{2 \eta_{p}} \boldsymbol{\sigma}-\nabla^{\mathrm{s}} \boldsymbol{u}+\frac{\lambda}{2 \eta_{p}}\left(\boldsymbol{a} \cdot \nabla \boldsymbol{\sigma}-\boldsymbol{\sigma} \cdot \nabla \boldsymbol{a}-(\nabla \boldsymbol{a})^{T} \cdot \boldsymbol{\sigma}\right)
\end{array}\right),
$$

we may write (2.1)-(2.3) as

$$
\mathcal{L}_{\mathrm{st}}(\boldsymbol{U})=\boldsymbol{F}
$$

The simplest boundary condition $\boldsymbol{u}=\mathbf{0}$ on $\partial \Omega$ will be considered throughout. 


\subsection{The log-conformation reformulation}

The log-conformation reformulation basically consists of a change of variables in terms of the matrix-logarithm of the conformation tensor $\tau$, defined from the expression

$$
\boldsymbol{\sigma}=\frac{\eta_{p}}{\lambda}(\boldsymbol{\tau}-\boldsymbol{I})
$$

The conformation tensor is replaced by a new variable $\boldsymbol{\psi}=\log (\boldsymbol{\tau})$, which can be calculated through an eigenvalue computation that rotates the $\boldsymbol{\tau}$ tensor into its main principle axes and can be expressed as $\boldsymbol{\psi}=\boldsymbol{R}_{\tau} \log (\boldsymbol{\Lambda}) \boldsymbol{R}_{\tau}^{T}$ because $\boldsymbol{\tau}$ is a symmetric positive definite tensor and therefore it can always be diagonalized. In the expression introduced, $\boldsymbol{\Lambda}$ is a diagonal matrix with the eigenvalues of $\boldsymbol{\tau}$, and $\boldsymbol{R}_{\tau}$ is the orthogonal matrix of the eigenvectors of $\boldsymbol{\tau}$. However, we have considered a modification when the conformation tensor is defined, with the aim of allowing $\lambda=0$, i.e., the Newtonian behavior. To this end, we introduce a modified relaxation-time parameter $\lambda_{0}(\lambda)$, which can be defined as $\lambda_{0}=\max \left\{k \lambda, \lambda_{0, \min }\right\}, k>0$ being a constant and $\lambda_{0, \min }$ a given threshold. So, if $k=1$ and $\lambda_{0, \min }=0$, the original change of variables proposed in [14] is recovered. It is worth to remark that in the numerical experiments we have found useful to take $k$ small, so that $\lambda_{0}<\lambda$; this has allowed us to obtain converged solutions that we have not been able to get for $k=1$; see [28].

Thus, we compute the modified conformation tensor, still denoted by $\tau$, from the expression

$$
\boldsymbol{\sigma}=\frac{\eta_{p}}{\lambda_{0}}(\boldsymbol{\tau}-\boldsymbol{I})
$$

Again, the conformation tensor $\boldsymbol{\tau}$ must be written as $\boldsymbol{\tau}=\exp (\boldsymbol{\psi})$ in the standard viscoelastic formulation detailed above (2.1)-(2.3).

To motivate the linearized problem to be analyzed, let us consider a Newton-Raphson linearization. Assume $\hat{\boldsymbol{\psi}}$ is the result obtained from a certain iteration and we need to compute the correction $\delta \hat{\boldsymbol{\psi}}=\boldsymbol{\psi}-\hat{\boldsymbol{\psi}}$. If this correction is small, we may approximate

$$
\exp (\boldsymbol{\psi})=\exp (\hat{\boldsymbol{\psi}}) \cdot \exp (\delta \boldsymbol{\psi}) \approx \exp (\hat{\boldsymbol{\psi}}) \cdot(\boldsymbol{I}+\delta \boldsymbol{\psi})=\exp (\hat{\boldsymbol{\psi}}) \cdot \boldsymbol{\psi}+\exp (\hat{\boldsymbol{\psi}}) \cdot(\boldsymbol{I}-\hat{\boldsymbol{\psi}}) .
$$

Since we consider $\exp (\hat{\boldsymbol{\psi}})$ and $\hat{\boldsymbol{\psi}}$ known, we can denote these tensors as $\boldsymbol{E}=\exp (\hat{\boldsymbol{\psi}})$ and $\boldsymbol{S}=\hat{\boldsymbol{\psi}}$, respectively, and introduce $\boldsymbol{R}=\boldsymbol{E} \cdot \boldsymbol{S}-\boldsymbol{E}$. The linearized equations of the log-conformation formulation are now expressed as follows:

$$
\begin{array}{r}
-\frac{\eta_{0}(1-\beta)}{\lambda_{0}} \nabla \cdot(\boldsymbol{E} \cdot \boldsymbol{\psi}-\boldsymbol{R})-2 \beta \eta_{0} \nabla \cdot\left(\nabla^{\mathrm{s}} \boldsymbol{u}\right)+\rho \boldsymbol{a} \cdot \nabla \boldsymbol{u}+\nabla p=\boldsymbol{f}, \\
\nabla \cdot \boldsymbol{u}=0, \\
\frac{1}{2 \lambda_{0}}(\boldsymbol{E} \cdot \boldsymbol{\psi}-\boldsymbol{R}-\boldsymbol{I})-\nabla^{\mathrm{s}} \boldsymbol{u} \\
+\frac{\lambda}{2 \lambda_{0}}\left(\boldsymbol{a} \cdot \nabla(\boldsymbol{E} \cdot \boldsymbol{\psi}-\boldsymbol{R})-(\boldsymbol{E} \cdot \boldsymbol{\psi}-\boldsymbol{R}) \cdot \nabla \boldsymbol{a}-(\nabla \boldsymbol{a})^{T} \cdot(\boldsymbol{E} \cdot \boldsymbol{\psi}-\boldsymbol{R})+2 \nabla^{\mathrm{s}} \boldsymbol{u}\right)=\mathbf{0},
\end{array}
$$

where the unknowns are the velocity, the pressure, and tensor $\boldsymbol{\psi}$. Note the presence of the last term $2 \nabla^{\mathrm{s}} \boldsymbol{u}$, which will have a crucial role in the dependence of the error estimate to be obtained with the Weissenberg number.

Let us introduce some notation, useful in the next subsections. Calling now $\boldsymbol{U}=[\boldsymbol{u}, p, \boldsymbol{\psi}]$ and defining

$$
\mathcal{L}(\boldsymbol{U}):=\left(\begin{array}{c}
-\frac{\eta_{p}}{\lambda_{0}} \nabla \cdot(\boldsymbol{E} \cdot \boldsymbol{\psi})-2 \beta \eta_{0} \nabla \cdot\left(\nabla^{\mathrm{s}} \boldsymbol{u}\right)+\rho \boldsymbol{a} \cdot \nabla \boldsymbol{u}+\nabla p \\
\nabla \cdot \boldsymbol{u} \\
\frac{1}{2 \lambda_{0}} \boldsymbol{E} \cdot \boldsymbol{\psi}-\nabla^{\mathrm{s}} \boldsymbol{u}+\frac{\lambda}{2 \lambda_{0}}\left(\boldsymbol{a} \cdot \nabla(\boldsymbol{E} \cdot \boldsymbol{\psi})-(\boldsymbol{E} \cdot \boldsymbol{\psi}) \cdot \nabla \boldsymbol{a}-(\nabla \boldsymbol{a})^{T} \cdot(\boldsymbol{E} \cdot \boldsymbol{\psi})+2 \nabla^{\mathrm{s}} \boldsymbol{u}\right)
\end{array}\right)
$$




$$
\boldsymbol{F}:=\left(\begin{array}{c}
\boldsymbol{f}-\frac{\eta_{p}}{\lambda_{0}} \nabla \cdot \boldsymbol{R} \\
0 \\
\frac{1}{2 \lambda_{0}}(\boldsymbol{I}+\boldsymbol{R})+\frac{\lambda}{2 \lambda_{0}}\left(\boldsymbol{a} \cdot \nabla \boldsymbol{R}-\boldsymbol{R} \cdot \nabla \boldsymbol{a}-(\nabla \boldsymbol{a})^{T} \cdot \boldsymbol{R}\right)
\end{array}\right)=:\left(\begin{array}{c}
\boldsymbol{f}_{u} \\
0 \\
\boldsymbol{f}_{\psi}
\end{array}\right)
$$

we may write $(2.7)-(2.9)$ as

$$
\mathcal{L}(\boldsymbol{U})=\boldsymbol{F},
$$

which again needs to be supplied with the boundary condition $\boldsymbol{u}=\mathbf{0}$ on $\partial \Omega$.

\subsection{Variational formulation}

In order to write the weak form of the problem, let us introduce some notation. The space of square integrable functions in a domain $\omega$ is denoted by $L^{2}(\omega)$, and the space of functions whose distributional derivatives of order up to $m \geq 0$ (integer) belong to $L^{2}(\omega)$ is denoted by $H^{m}(\omega)$. Essentially bounded functions in $\omega$ are denoted by $L^{\infty}(\omega)$.

The space $H_{0}^{1}(\omega)$ is made up of functions in $H^{1}(\omega)$ vanishing on $\partial \omega$. The topological dual of $H_{0}^{1}(\omega)$ is denoted by $H^{-1}(\Omega)$, the duality pairing being $\langle\cdot, \cdot\rangle$. The $L^{2}$ inner product in $\omega$ (for scalars, vectors and tensors) is denoted by $(\cdot, \cdot)_{\omega}$ and the integral over $\omega$ of the product of two general functions is written as $\langle\cdot, \cdot\rangle_{\omega}$, the subscript being omitted when $\omega=\Omega$. The norm in a space $X$ is denoted by $\|\cdot\|_{X}$, except when $X=L^{2}(\Omega)$, case in which the subscript is omitted.

Using this notation, the velocity and pressure spaces for the continuous problem are $\mathcal{V}=H_{0}^{1}(\Omega)^{d}$ and $\mathcal{Q}=L^{2}(\Omega) / \mathbb{R}$, and the space for the tensor $\boldsymbol{\psi}$ is denoted by $\boldsymbol{\Upsilon} \subset L^{2}(\Omega)^{d \times d}$, with appropriate regularity to make the following weak form well defined. This weak form consists in finding $\boldsymbol{U}=[\boldsymbol{u}, p, \boldsymbol{\psi}] \in \mathcal{X}:=\mathcal{V} \times \mathcal{Q} \times \boldsymbol{\Upsilon}$ such that

$$
\begin{aligned}
\frac{\eta_{p}}{\lambda_{0}}\left(\boldsymbol{E} \cdot \boldsymbol{\psi}, \nabla^{\mathrm{s}} \boldsymbol{v}\right)+2\left(\beta \eta_{0} \nabla^{\mathrm{s}} \boldsymbol{u}, \nabla^{\mathrm{s}} \boldsymbol{v}\right)+\langle\rho \boldsymbol{a} \cdot \nabla \boldsymbol{u}, \boldsymbol{v}\rangle-(p, \nabla \cdot \boldsymbol{v}) & =\langle\boldsymbol{f}, \boldsymbol{v}\rangle+\frac{\eta_{p}}{\lambda_{0}}\left(\boldsymbol{R}, \nabla^{\mathrm{s}} \boldsymbol{v}\right), \\
(q, \nabla \cdot \boldsymbol{u}) & =0, \\
\frac{1}{2 \lambda_{0}}(\boldsymbol{E} \cdot \boldsymbol{\psi}, \boldsymbol{\chi})-\left(\nabla^{\mathrm{s}} \boldsymbol{u}, \boldsymbol{\chi}\right)+\frac{\lambda}{2 \lambda_{0}}(\boldsymbol{a} \cdot \nabla(\boldsymbol{E} \cdot \boldsymbol{\psi}), \boldsymbol{\chi}) & \\
+\frac{\lambda}{2 \lambda_{0}}\left(-\boldsymbol{E} \cdot \boldsymbol{\psi} \cdot \nabla \boldsymbol{a}-(\nabla \boldsymbol{a})^{T} \cdot \boldsymbol{E} \cdot \boldsymbol{\psi}+2 \nabla^{\mathrm{s}} \boldsymbol{u}, \boldsymbol{\chi}\right) & =\frac{1}{2 \lambda_{0}}(\boldsymbol{I}+\boldsymbol{R}, \boldsymbol{\chi})+\frac{\lambda}{2 \lambda_{0}}(\boldsymbol{a} \cdot \nabla \boldsymbol{R}, \boldsymbol{\chi}) \\
& +\frac{\lambda}{2 \lambda_{0}}\left(-\boldsymbol{R} \cdot \nabla \boldsymbol{a}-(\nabla \boldsymbol{a})^{T} \cdot \boldsymbol{R}, \boldsymbol{\chi}\right),
\end{aligned}
$$

for all $\boldsymbol{V}=[\boldsymbol{v}, q, \boldsymbol{\chi}] \in \mathcal{X}$, where it is assumed that $\boldsymbol{f}, \boldsymbol{R}$ and $\boldsymbol{E}$ are such that the known terms are well defined. In compact form, the problem can be written as:

$$
B(\boldsymbol{U}, \boldsymbol{V})=L(\boldsymbol{V})
$$

where

$$
\begin{aligned}
B(\boldsymbol{U}, \boldsymbol{V})= & \frac{\eta_{p}}{\lambda_{0}}\left(\boldsymbol{E} \cdot \boldsymbol{\psi}, \nabla^{\mathrm{s}} \boldsymbol{v}\right)+2\left(\beta \eta_{0} \nabla^{\mathrm{s}} \boldsymbol{u}, \nabla^{\mathrm{s}} \boldsymbol{v}\right)+\langle\rho \boldsymbol{a} \cdot \nabla \boldsymbol{u}, \boldsymbol{v}\rangle-(p, \nabla \cdot \boldsymbol{v}) \\
& +(\nabla \cdot \boldsymbol{u}, q)+\frac{1}{2 \lambda_{0}}(\boldsymbol{E} \cdot \boldsymbol{\psi}, \boldsymbol{\chi})-\left(\nabla^{\mathrm{s}} \boldsymbol{u}, \boldsymbol{\chi}\right) \\
& +\frac{\lambda}{2 \lambda_{0}}\left(\boldsymbol{a} \cdot \nabla(\boldsymbol{E} \cdot \boldsymbol{\psi})-\boldsymbol{E} \cdot \boldsymbol{\psi} \cdot \nabla \boldsymbol{a}-(\nabla \boldsymbol{a})^{T} \cdot \boldsymbol{E} \cdot \boldsymbol{\psi}+2 \nabla^{\mathrm{s}} \boldsymbol{u}, \boldsymbol{\chi}\right), \\
L(\boldsymbol{V})= & \langle\boldsymbol{f}, \boldsymbol{v}\rangle+\frac{\eta_{p}}{\lambda_{0}}\left(\boldsymbol{R}, \nabla^{\mathrm{s}} \boldsymbol{v}\right)+\frac{1}{2 \lambda_{0}}(\boldsymbol{I}+\boldsymbol{R}, \boldsymbol{\chi})+\frac{\lambda}{2 \lambda_{0}}(\boldsymbol{a} \cdot \nabla \boldsymbol{R}, \boldsymbol{\chi}) \\
& +\frac{\lambda}{2 \lambda_{0}}\left(-\boldsymbol{R} \cdot \nabla \boldsymbol{a}-(\nabla \boldsymbol{a})^{T} \cdot \boldsymbol{R}, \boldsymbol{\chi}\right) .
\end{aligned}
$$


Note that from the physical point of view the test function $\chi$ is a stress, whereas the dimensionless unknown $\psi$ is the logarithm of the conformation tensor. We could also have used a test function for the constitutive equation of the form $\frac{\eta_{p}}{\lambda_{0}} \exp (\chi)$, where now $\chi$ would be dimensionless. This would simplify the analysis (some stability would follow taking $\chi=\boldsymbol{\psi}$ ), but complicate significantly the FE approximations described below. Note that, strictly speaking, the space of stress test functions could be taken as the $L^{2}$ projection onto $L^{2}(\Omega)^{d \times d}$ of functions of the form $\boldsymbol{E} \cdot \boldsymbol{\varphi}$ properly scaled, for example by a factor $\frac{\eta_{p}}{\lambda_{0}}$, with $\varphi$ belonging to the space of trial solutions.

We will not analyze the continuous problem (2.17), but simply assume that there exists a solution that is smooth enough. As for the standard formulation, this requires $\lambda$ to be small enough and, in the case of the linearization we consider, the following condition on the velocity $\boldsymbol{a}$ which will also be needed in the discrete problem:

Assumption H1 $\quad \boldsymbol{a} \in \mathcal{C}^{0}(\bar{\Omega})^{d}, \nabla \cdot \boldsymbol{a}=0, \boldsymbol{a}$ and $\nabla \boldsymbol{a}$ have components in $L^{\infty}(\Omega)$.

$\boldsymbol{E}$ and $\boldsymbol{R}$ have components in $L^{\infty}(\Omega)$.

$\boldsymbol{E}$ is invertible with a bounded inverse.

\subsection{Stability of the Galerkin finite element discretization}

The standard Galerkin approximation for the variational problem, which has been established in (2.17), is described next. Let $\mathcal{T}_{h}=\{K\}$ be a FE partition of the domain $\Omega$. The diameter of an element $K \in \mathcal{T}_{h}$ is denoted by $h_{K}$ and the diameter of the partition is defined as $h=\max \left\{h_{K} \mid K \in \mathcal{T}_{h}\right\}$. For simplicity, we will consider quasi-uniform partitions in the following. The $L^{2}$ norm in an element $K$ will be denoted by $\|\cdot\|_{K}$.

From $\mathcal{T}_{h}$ we may construct conforming FE spaces for the velocity, the pressure and the elastic stress, $\mathcal{V}_{h} \subset \mathcal{V}$, $\mathcal{Q}_{h} \subset \mathcal{Q}, \Upsilon_{h} \subset \boldsymbol{\Upsilon}$, respectively. Although any conforming approximation could be considered using the approach to be described, and this means that pressures and stresses could be discontinuous (see [6]), for conciseness we will restrict to continuous interpolations for these fields.

The condition that the convective derivative of the stress be square integrable will follow from $\mathrm{H} 1$ and choosing the stresses continuous, for example. Calling $\mathcal{X}_{h}:=\mathcal{V}_{h} \times \mathcal{Q}_{h} \times \boldsymbol{\Upsilon}_{h}$, the Galerkin FE approximation of the problem consists in finding $\boldsymbol{U}_{h} \in \mathcal{X}_{h}$, such that:

$$
B_{\psi}\left(\boldsymbol{U}_{h}, \boldsymbol{V}_{h}\right)=L\left(\boldsymbol{V}_{h}\right),
$$

for all $\boldsymbol{V}_{h}=\left[\boldsymbol{v}_{h}, q_{h}, \boldsymbol{\chi}_{h}\right] \in \mathcal{X}_{h}$, where $B_{\psi}$ is obtained from $B$ given in (2.18) replacing $\boldsymbol{E} \cdot \boldsymbol{\psi}_{h}$ by $P_{\psi}\left(\boldsymbol{E} \cdot \boldsymbol{\psi}_{h}\right)$, where $P_{\psi}$ is the $L^{2}$ projection onto $\boldsymbol{\Upsilon}_{h}$.

As in the standard formulation, problem (2.20) lacks stability unless appropriate inf-sup conditions hold. Likewise, convective terms are not bounded, and these may dominate those that can be controlled.

\section{Stabilized Finite Element Method}

VMS methods consist in the splitting of the unknown $\boldsymbol{U}$ in a component $\boldsymbol{U}_{h}$, which can be resolved by the FE space, and the remainder $\tilde{\boldsymbol{U}}$, that will be called sub-grid scale. The framework is based on the work by Hughes et al. [20]. In the context of a three field formulation for flow problems, see [5,11]. The sub-grid scale needs to be approximated in a simple manner, with the goal of capturing its effect and yielding a stable formulation. The particular approach we follow and the resulting stabilized finite element method are described next.

\subsection{Residual based stabilization}

The problem we wish to approximate is (2.12) in differential form and (2.17) in variational form. After introducing the subscale decomposition and integrating by parts, it can be readily checked (see [12]) that the VMS method leads to the problem of finding $\boldsymbol{U}_{h} \in \boldsymbol{\mathcal { X }}_{h}$ such that

$$
B_{\psi}\left(\boldsymbol{U}_{h}, \boldsymbol{V}_{h}\right)+\sum_{K}\left\langle\tilde{\boldsymbol{U}}, \mathcal{L}_{\mathrm{st}}^{*}\left(\boldsymbol{V}_{h}\right)\right\rangle_{K}=L\left(\boldsymbol{V}_{h}\right)
$$


for all $\boldsymbol{V}_{h} \in \mathcal{X}_{h}$, where $\mathcal{L}_{\mathrm{st}}^{*}$ is the formal adjoint operator of $\mathcal{L}_{\text {st }}$ and $\tilde{\boldsymbol{U}}$ is the sub-grid scale that needs to be approximated. $\mathcal{L}_{\mathrm{st}}^{*}$ is given by

$$
\mathcal{L}_{\mathrm{st}}^{*}(\boldsymbol{V}):=\left(\begin{array}{c}
\nabla \cdot \boldsymbol{\chi}-2 \eta_{e} \nabla \cdot\left(\nabla^{\mathrm{s}} \boldsymbol{v}\right)-\rho \boldsymbol{a} \cdot \nabla \boldsymbol{v}-\nabla q \\
-\nabla \cdot \boldsymbol{v} \\
\frac{1}{2 \eta_{p}} \boldsymbol{\chi}+\nabla^{\mathrm{s}} \boldsymbol{v}-\frac{\lambda}{2 \eta_{p}}\left(\boldsymbol{a} \cdot \nabla \boldsymbol{\chi}+\boldsymbol{\chi} \cdot(\nabla \boldsymbol{a})^{T}+\nabla \boldsymbol{a} \cdot \boldsymbol{\chi}\right)
\end{array}\right) .
$$

The fact that the operator that appears after integration by parts is the adjoint of $\mathcal{L}_{\text {st }}$ and not of $\mathcal{L}$ is simply due to the way in which we have written the equations, changing variables in the stress but not in the stress test function.

Taking $\tilde{P}$ as the $L^{2}$ projection onto the space of sub-grid scales, the approximation we consider for the sub-grid scales within each element is

$$
\tilde{\boldsymbol{U}}=\boldsymbol{\alpha} \tilde{P}\left[\boldsymbol{F}-\mathcal{L}\left(\boldsymbol{U}_{h}\right)\right]
$$

where $\boldsymbol{\alpha}$ is a diagonal matrix of the form $\boldsymbol{\alpha}=\operatorname{diag}\left(\alpha_{u} \boldsymbol{I}_{d}, \alpha_{p}, \alpha_{\psi} \boldsymbol{I}_{d \times d}\right)$ with $\boldsymbol{I}_{d}$ the identity on vectors of $\mathbb{R}^{d}$, $\boldsymbol{I}_{d \times d}$ the identity on second order tensor and the parameters $\alpha_{u}, \alpha_{p}$ and $\alpha_{\psi}$ computed as

$$
\begin{aligned}
\alpha_{u} & =\left[c_{1} \frac{\eta_{0}}{h^{2}}+c_{2} \frac{\rho|\boldsymbol{a}|}{h}\right]^{-1}, \\
\alpha_{p} & =\frac{h^{2}}{c_{1} \alpha_{1}}, \\
\alpha_{\psi} & =\left[c_{3} \frac{1}{2 \eta_{p}}+c_{4}\left(\frac{\lambda}{2 \eta_{p}} \frac{|\boldsymbol{a}|}{h}+\frac{\lambda}{\eta_{p}}|\nabla \boldsymbol{a}|\right)\right]^{-1},
\end{aligned}
$$

where $|\boldsymbol{a}|$ is the Euclidean norm while $|\nabla \boldsymbol{a}|$ is the Frobenius norm. The dimensionless constants $c_{i}, i=1, \ldots, 4$ are algorithmic parameters in the formulation, which have to be of order one [11].

\subsection{Split-OSS}

The Orthogonal Sub-grid Scale (OSS) stabilization $[8,9]$ consists in taking $\tilde{P}=P_{h}^{\perp}$, where $P_{h}$ is the $L^{2}$ projection onto $\mathcal{X}_{h}$. In this case, we can design a simplified method, which consists in keeping only the terms of the form one operator term applied to the unknown by the same operator term applied to the test function, thus neglecting the products of different operators [10]. We call Split-OSS method the resulting stabilized formulation.

Following the considerations made in [7] for the construction of the Split-OSS method for the traditional viscoelastic formulation, the modified method we propose for the log-conformation reformulation is: find $\boldsymbol{U}_{h} \in$ $\mathcal{X}_{h}$ such that

$$
B_{\text {stab }}\left(\boldsymbol{U}_{h}, \boldsymbol{V}_{h}\right)=B_{\psi}\left(\boldsymbol{U}_{h}, \boldsymbol{V}_{h}\right)+B^{*}\left(\boldsymbol{U}_{h}, \boldsymbol{V}_{h}\right)=L\left(\boldsymbol{V}_{h}\right)
$$

for all $\boldsymbol{V}_{h} \in \mathcal{X}_{h}$, where $B^{*}\left(\boldsymbol{U}_{h}, \boldsymbol{V}_{h}\right)$ represents the stabilizing part of the model, defined as

$$
B^{*}\left(\boldsymbol{U}_{h}, \boldsymbol{V}_{h}\right)=S_{1}^{\perp}\left(\boldsymbol{U}_{h}, \boldsymbol{V}_{h}\right)+S_{2}^{\perp}\left(\boldsymbol{U}_{h}, \boldsymbol{V}_{h}\right)+S_{3}^{\perp}\left(\boldsymbol{u}_{h} ; \boldsymbol{U}_{h}, \boldsymbol{V}_{h}\right)
$$

where

$$
\begin{aligned}
S_{1}^{\perp}\left(\boldsymbol{U}_{h}, \boldsymbol{V}_{h}\right)= & \sum_{K} \alpha_{u}\left\langle P_{u}^{\perp}\left[-\frac{\eta_{p}}{\lambda_{0}} \nabla \cdot P_{\psi}\left[\boldsymbol{E} \cdot \boldsymbol{\psi}_{h}\right]\right],-\nabla \cdot \boldsymbol{\chi}_{h}\right\rangle_{K} \\
& +\sum_{K} \alpha_{u}\left\langle P_{u}^{\perp}\left[\nabla p_{h}\right], \nabla q_{h}\right\rangle_{K}+\sum_{K} \alpha_{u}\left\langle P_{u}^{\perp}\left[\rho \boldsymbol{a} \cdot \nabla \boldsymbol{u}_{h}\right], \rho \boldsymbol{a} \cdot \nabla \boldsymbol{v}_{h}\right\rangle_{K}, \\
S_{2}^{\perp}\left(\boldsymbol{U}_{h}, \boldsymbol{V}_{h}\right)= & \sum_{K} \alpha_{p}\left\langle P_{p}^{\perp}\left[\nabla \cdot \boldsymbol{u}_{h}\right], \nabla \cdot \boldsymbol{v}_{h}\right\rangle_{K}, \\
S_{3}^{\perp}\left(\boldsymbol{U}_{h}, \boldsymbol{V}_{h}\right)= & \sum_{K} \alpha_{\psi}\left\langle P_{\psi}^{\perp}\left[\boldsymbol{R}_{\psi}\right],-\nabla^{\mathrm{s}} \boldsymbol{v}_{h}+\frac{\lambda}{2 \eta_{p}}\left(\boldsymbol{a} \cdot \nabla \boldsymbol{\chi}_{h}+\boldsymbol{\chi}_{h} \cdot(\nabla \boldsymbol{a})^{T}+\nabla \boldsymbol{a} \cdot \boldsymbol{\chi}_{h}\right)\right\rangle_{K},
\end{aligned}
$$


and where $\boldsymbol{R}_{\psi}$ is the residual of the constitutive equation

$$
\boldsymbol{R}_{\psi}=-\nabla^{\mathrm{s}} \boldsymbol{u}_{h}+\frac{\lambda}{2 \lambda_{0}}\left(\boldsymbol{a} \cdot \nabla P_{\psi}\left[\boldsymbol{E} \cdot \boldsymbol{\psi}_{h}\right]-P_{\psi}\left[\boldsymbol{E} \cdot \boldsymbol{\psi}_{h}\right] \cdot \nabla \boldsymbol{a}-(\nabla \boldsymbol{a})^{T} \cdot P_{\psi}\left[\boldsymbol{E} \cdot \boldsymbol{\psi}_{h}\right]+2 \nabla^{\mathrm{s}} \boldsymbol{u}_{h}\right) .
$$

The $L^{2}$ projections onto the FE spaces for velocity (without boundary conditions), pressure and stress have respectively been denoted by $P_{u}, P_{p}$ and, as already mentioned, $P_{\psi}$. The projection onto the velocity space with boundary conditions will be denoted $P_{u, 0}$.

The method is a mix of an orthogonal term-by-term formulation for the momentum equation and continuity equation and a residual-based formulation for the constitutive equation. For smooth solutions, both have an optimal convergence rate in $h$. However, in problems where the solution has strong gradients, we have found (3.7) more robust, similarly to what it is explained in [7]. For a detailed motivation and numerical experimentation using this method, see [28].

In the numerical analysis below we will also use the notation

$$
P_{\psi}\left[\boldsymbol{E} \cdot \boldsymbol{\psi}_{h}\right] \cdot \nabla \boldsymbol{a}+(\nabla \boldsymbol{a})^{T} \cdot P_{\psi}\left[\boldsymbol{E} \cdot \boldsymbol{\psi}_{h}\right]=\dot{\boldsymbol{\psi}}_{h}^{*}+\dot{\boldsymbol{\psi}}_{h}^{* *},
$$

and

$$
P_{\psi}\left[\boldsymbol{E} \cdot \boldsymbol{\psi}_{h}\right] \cdot(\nabla \boldsymbol{a})^{T}+\nabla \boldsymbol{a} \cdot P_{\psi}\left[\boldsymbol{E} \cdot \boldsymbol{\psi}_{h}\right]=\dot{\boldsymbol{\psi}}_{h}^{*}-\dot{\boldsymbol{\psi}}_{h}^{*},
$$

where $\dot{\boldsymbol{\psi}}_{h}^{*}=P_{\psi}\left[\boldsymbol{E} \cdot \boldsymbol{\psi}_{h}\right] \cdot \nabla^{\mathrm{s}} \boldsymbol{a}+\nabla^{\mathrm{s}} \boldsymbol{a} \cdot P_{\psi}\left[\boldsymbol{E} \cdot \boldsymbol{\psi}_{h}\right]$ and $\dot{\boldsymbol{\psi}}_{h}^{* *}=P_{\psi}\left[\boldsymbol{E} \cdot \boldsymbol{\psi}_{h}\right] \cdot \nabla^{\mathrm{as}} \boldsymbol{a}-\nabla^{\mathrm{as}} \boldsymbol{a} \cdot P_{\psi}\left[\boldsymbol{E} \cdot \boldsymbol{\psi}_{h}\right]$. In these expressions, $\nabla^{\text {as }} \boldsymbol{a}$ represents the skew-symmetric part of the velocity gradient, given by

$$
\nabla^{\mathrm{as}} \boldsymbol{a}=\frac{1}{2}\left[\nabla \boldsymbol{a}-(\nabla \boldsymbol{a})^{T}\right] .
$$

\section{NumericAl ANALYSiS}

\subsection{Preliminaries}

We assume that there is a constant $c_{\text {inv }}$, independent of the mesh size $h$, such that

$$
\left\|\nabla v_{h}\right\|_{K} \leq c_{\mathrm{inv}} h^{-1}\left\|v_{h}\right\|_{K}
$$

for all FE functions $v_{h}$ defined on $K \in \mathcal{T}_{h}$, which can be either scalars, vectors or tensors. We will also make use of Korn's inequality, which holds for the conforming approximation that we consider:

$$
\left\|\boldsymbol{v}_{h}\right\|_{H^{1}(\Omega)}^{2} \leq c_{L}\left\|\nabla^{\mathrm{s}} \boldsymbol{v}_{h}\right\|^{2} \quad \text { with } \quad \boldsymbol{v}_{h}=\mathbf{0} \quad \text { on } \quad \partial \Omega
$$

$c_{L}>0$ being a constant. As usual, $C$ will denote a generic positive constant, possibly different at different occurrences. A fixed constant will be identified with a subscript.

We will need a condition on the interpolating spaces that holds in the case of equal order interpolations, and that can be written as $[6,11]$ :

Assumption H2 Given $\boldsymbol{a}, \boldsymbol{v}_{h} \in \mathcal{V}_{h}, q_{h} \in \mathcal{Q}_{h}, \boldsymbol{\psi}_{h} \in \boldsymbol{\Upsilon}_{h}$ and $\boldsymbol{z}_{h}:=\rho \boldsymbol{a} \cdot \nabla \boldsymbol{v}_{h}+\nabla q_{h}-\frac{\eta_{p}}{\lambda_{0}} \nabla \cdot P_{\psi}\left[\boldsymbol{E} \cdot \boldsymbol{\psi}_{h}\right]$, there holds $\left\|\boldsymbol{z}_{h}\right\| \leq c_{\mathrm{m}}\left(\left\|P_{u, 0}\left[\boldsymbol{z}_{h}\right]\right\|+\left\|P_{u}^{\perp}\left[\boldsymbol{z}_{h}\right]\right\|\right)$, for a constant $c_{\mathrm{m}}>0$.

For a piecewise linear velocity $\boldsymbol{a}$ this assumption is known to hold; here we assume that $\boldsymbol{a}$ is such that it is satisfied. Note that $c_{\mathrm{m}}$ may depend on the different components of $\boldsymbol{z}_{h}$, but not on its Euclidean norm. 


\subsection{Stability and convergence in a mesh-dependent norm}

The norm in which the results will be first presented is

$$
\begin{aligned}
\left\|\boldsymbol{V}_{h}\right\|_{W}^{2}= & 2 \eta_{e}\left\|\nabla^{s} \boldsymbol{v}_{h}\right\|^{2}+\frac{\eta_{p}}{\lambda_{0}^{2}}\left\|P_{\psi}\left[\boldsymbol{E} \cdot \boldsymbol{\varphi}_{h}\right]\right\|^{2}+\sum_{K} \alpha_{u}\left\|\rho \boldsymbol{a} \cdot \nabla \boldsymbol{v}_{h}+\nabla q_{h}-\frac{\eta_{p}}{\lambda_{0}} \nabla \cdot P_{\psi}\left[\boldsymbol{E} \cdot \boldsymbol{\varphi}_{h}\right]\right\|_{K}^{2} \\
& +\sum_{K} \alpha_{u}\left\|P_{u}^{\perp}\left[\rho \boldsymbol{a} \cdot \nabla \boldsymbol{v}_{h}\right]\right\|_{K}^{2}+\sum_{K} \alpha_{u}\left\|P_{u}^{\perp}\left[\nabla q_{h}\right]\right\|_{K}^{2}+\sum_{K} \alpha_{u}\left\|P_{u}^{\perp}\left[\frac{\eta_{p}}{\lambda_{0}} \nabla \cdot P_{\psi}\left[\boldsymbol{E} \cdot \boldsymbol{\varphi}_{h}\right]\right]\right\|_{K}^{2} \\
& +\sum_{K} \alpha_{p}\left\|\nabla \cdot \boldsymbol{v}_{h}\right\|_{K}^{2}+\sum_{K} \alpha_{\psi}\left\|\frac{\lambda}{2 \lambda_{0}}\left(\boldsymbol{a} \cdot \nabla P_{\psi}\left[\boldsymbol{E} \cdot \boldsymbol{\varphi}_{h}\right]-\dot{\boldsymbol{\varphi}}_{h}^{* *}\right)\right\|_{K}^{2}
\end{aligned}
$$

considering $\boldsymbol{V}_{h}=\left[\boldsymbol{v}_{h}, q_{h}, \boldsymbol{\varphi}_{h}\right] \in \mathcal{X}_{h}$ (note again that $\boldsymbol{\varphi}_{h}$ is dimensionless). This is clearly a norm for the homogeneous velocity boundary conditions considered, since if $\left\|\boldsymbol{V}_{h}\right\|_{W}=0, \boldsymbol{v}_{h}=\mathbf{0}$ because of the first term (using Körn's inequality), $P_{\psi}\left[\boldsymbol{E} \cdot \boldsymbol{\varphi}_{h}\right]=\mathbf{0}$ for $\eta_{p}>0$ because of the second term (and, in fact, $\boldsymbol{\varphi}_{h}=\mathbf{0}$ because of Assumption H3 stated later), and, finally, $q_{h}=0$ because of the third term and the definition of $\mathcal{Q}$.

To simplify the analysis, we shall consider that the stabilization parameters are constant, computed with the $L^{\infty}(\Omega)$ norm of the advection velocity and its gradient. The analysis of variable stabilization parameters, including non-uniform meshes, can be done using the techniques in [10].

The main stability result, which implies existence and uniqueness of discrete solutions, is the following:

Theorem 4.1 (Stability). Suppose that H1 and H2 hold. For $\lambda$ small enough compared to the rest of physical parameters, there is a constant $C>0$ such that

$$
\inf _{\boldsymbol{U}_{h} \in \mathcal{X}_{h}} \sup _{\boldsymbol{V}_{h} \in \mathcal{X}_{h}} \frac{B_{\text {stab }}\left(\boldsymbol{U}_{h}, \boldsymbol{V}_{h}\right)}{\left\|\boldsymbol{U}_{h}\right\|_{W}\left\|\boldsymbol{V}_{h}\right\|_{W}} \geq C,
$$

provided the constants $c_{i}, i=1, \ldots, 4$ defined in (3.4)-(3.6) are large enough.

Proof. Given $\boldsymbol{U}_{h} \in \boldsymbol{\mathcal { X }}_{h}$, consider $\boldsymbol{U}_{h 1}=\left[\lambda^{*} \boldsymbol{u}_{h}, \lambda^{*} p_{h}, \frac{\eta_{p}}{\lambda_{0}} P_{\psi}\left[\boldsymbol{E} \cdot \boldsymbol{\psi}_{h}\right]\right]$, where $\lambda^{*}=\frac{\lambda-\lambda_{0}}{\lambda_{0}}>0$. We assume that $\lambda>\lambda_{0, \text { min }}$, since the case of very small elasticity is easier to prove and it is not our focus. Now, using the skew symmetry of the convective terms (from Assumption H1), we obtain:

$$
\begin{aligned}
B_{\text {stab }}\left(\boldsymbol{U}_{h}, \boldsymbol{U}_{h 1}\right)= & B_{\psi}\left(\boldsymbol{U}_{h}, \boldsymbol{U}_{h 1}\right)+B^{*}\left(\boldsymbol{U}_{h}, \boldsymbol{U}_{h 1}\right) \\
= & \underbrace{\frac{\eta_{p}}{\lambda_{0}} \lambda^{*}\left(P_{\psi}\left[\boldsymbol{E} \cdot \boldsymbol{\psi}_{h}\right], \nabla^{\mathrm{s}} \boldsymbol{u}_{h}\right)}_{(1)}+2 \eta_{e} \lambda^{*}\left(\nabla^{\mathrm{s}} \boldsymbol{u}_{h}, \nabla^{\mathrm{s}} \boldsymbol{u}_{h}\right) \\
& +\frac{1}{2 \lambda_{0}} \frac{\eta_{p}}{\lambda_{0}}\left(P_{\psi}\left[\boldsymbol{E} \cdot \boldsymbol{\psi}_{h}\right], P_{\psi}\left[\boldsymbol{E} \cdot \boldsymbol{\psi}_{h}\right]\right) \underbrace{\frac{\eta_{p}}{\lambda_{0}}\left(\nabla^{\mathrm{s}} \boldsymbol{u}_{h}, P_{\psi}\left[\boldsymbol{E} \cdot \boldsymbol{\psi}_{h}\right]\right)}_{(1)} \\
& +\frac{\lambda}{2 \lambda_{0}} \frac{\eta_{p}}{\lambda_{0}}(\underbrace{-P_{\psi}\left[\boldsymbol{E} \cdot \boldsymbol{\psi}_{h}\right] \cdot \nabla \boldsymbol{a}-(\nabla \boldsymbol{a})^{T} \cdot P_{\psi}\left[\boldsymbol{E} \cdot \boldsymbol{\psi}_{h}\right]}_{(2)}+\underbrace{2 \nabla^{\mathrm{s}} \boldsymbol{u}_{h}}_{(1)}, P_{\psi}\left[\boldsymbol{E} \cdot \boldsymbol{\psi}_{h}\right]) \\
& +\sum_{K} \alpha_{u}\left\langle P_{u}^{\perp}\left[\frac{\eta_{p}}{\lambda_{0}} \nabla \cdot P_{\psi}\left[\boldsymbol{E} \cdot \boldsymbol{\psi}_{h}\right]\right], \frac{\eta_{p}}{\lambda_{0}} \nabla \cdot P_{\psi}\left[\boldsymbol{E} \cdot \boldsymbol{\psi}_{h}\right]\right\rangle \\
& +\sum_{K} \alpha_{u} \lambda^{*}\left\langle P_{u}^{\perp}\left[\nabla p_{h}\right], \nabla p_{h}\right\rangle+\sum_{K} \alpha_{u} \lambda^{*}\left\langle P_{u}^{\perp}\left[\rho \boldsymbol{a} \cdot \nabla \boldsymbol{u}_{h}\right], \rho \boldsymbol{a} \cdot \nabla \boldsymbol{u}_{h}\right\rangle
\end{aligned}
$$




$$
\begin{aligned}
& +\sum_{K} \alpha_{p} \lambda^{*}\left\langle P_{p}^{\perp}\left[\nabla \cdot \boldsymbol{u}_{h}\right], \nabla \cdot \boldsymbol{u}_{h}\right\rangle \\
& +\sum_{K} \alpha_{\psi}\left\langle P_{\psi}^{\perp}\left[\lambda^{*} \nabla^{\mathrm{s}} \boldsymbol{u}_{h}+\frac{\lambda}{2 \lambda_{0}}\left(\boldsymbol{a} \cdot \nabla P_{\psi}\left[\boldsymbol{E} \cdot \boldsymbol{\psi}_{h}\right]-\dot{\boldsymbol{\psi}}_{h}^{*}-\dot{\boldsymbol{\psi}}_{h}^{* *}\right)\right],\right. \\
& \underbrace{\left.-\lambda^{*} \nabla^{\mathrm{s}} \boldsymbol{u}_{h}+\frac{\lambda}{2 \lambda_{0}}\left(\boldsymbol{a} \cdot \nabla P_{\psi}\left[\boldsymbol{E} \cdot \boldsymbol{\psi}_{h}\right]+\dot{\boldsymbol{\psi}}_{h}^{*}-\dot{\boldsymbol{\psi}}_{h}^{* *}\right)\right\rangle} .
\end{aligned}
$$

Let us bound the terms in this expression. From now on, $\varepsilon$ with a subscript will denote a constant resulting from Young's inequality, which will be repeatedly used together with Schwarz's inequality. One can easily obtain:

$$
\begin{aligned}
(1) \geq & -2 \eta_{p} \lambda^{*}\left[\frac{1}{2 \varepsilon_{0} \lambda_{0}^{2}}\left\|P_{\psi}\left[\boldsymbol{E} \cdot \boldsymbol{\psi}_{h}\right]\right\|^{2}+\frac{\varepsilon_{0}}{2}\left\|\nabla^{\mathrm{s}} \boldsymbol{u}_{h}\right\|^{2}\right] \\
(2) \geq & -\frac{\lambda \eta_{p}}{\lambda_{0}^{2}}\|\nabla \boldsymbol{a}\|_{L^{\infty}(\Omega)}\left\|P_{\psi}\left[\boldsymbol{E} \cdot \boldsymbol{\psi}_{h}\right]\right\|^{2}, \\
(3) \geq & -\left(\lambda^{*}\right)^{2} \sum_{K} \alpha_{\psi}\left\|\nabla^{\mathrm{s}} \boldsymbol{u}_{h}\right\|_{K}^{2}+\sum_{K} \alpha_{\psi}\left(\frac{\lambda}{2 \lambda_{0}}\right)^{2}\left\|P_{\psi}^{\perp}\left[\boldsymbol{a} \cdot \nabla P_{\psi}\left[\boldsymbol{E} \cdot \boldsymbol{\psi}_{h}\right]-\dot{\boldsymbol{\psi}}_{h}^{* *}\right]\right\|_{K}^{2} \\
& -\sum_{K} \alpha_{\psi}\left(\frac{\lambda}{2 \lambda_{0}}\right)^{2} 4\left\|\nabla^{\mathrm{s}} \boldsymbol{a}\right\|_{L^{\infty}(K)}^{2}\left\|P_{\psi}\left[\boldsymbol{E} \cdot \boldsymbol{\psi}_{h}\right]\right\|_{K}^{2},
\end{aligned}
$$

from where

$$
\begin{aligned}
B_{\text {stab }}\left(\boldsymbol{U}_{h}, \boldsymbol{U}_{h 1}\right) \geq & \sum_{K}\left(2 \eta_{e} \lambda^{*}-\eta_{p} \lambda^{*} \varepsilon_{0}-\left(\lambda^{*}\right)^{2} \alpha_{\psi}\right)\left\|\nabla^{\mathrm{s}} \boldsymbol{u}_{h}\right\|_{K}^{2} \\
& +\sum_{K}\left[\frac{\eta_{p}}{\lambda_{0}^{2}}\left(1-\frac{\lambda^{*}}{\varepsilon_{0}}-\lambda\|\nabla \boldsymbol{a}\|_{L^{\infty}(K)}\right)-\alpha_{\psi}\left(\frac{\lambda}{2 \lambda_{0}}\right)^{2} 4\left\|\nabla^{\mathrm{s}} \boldsymbol{a}\right\|_{L^{\infty}(K)}^{2}\right]\left\|P_{\psi}\left[\boldsymbol{E} \cdot \boldsymbol{\psi}_{h}\right]\right\|_{K}^{2} \\
& +\sum_{K} \alpha_{u}\left\|P_{u}^{\perp}\left[\frac{\eta_{p}}{\lambda_{0}} \nabla \cdot P_{\psi}\left[\boldsymbol{E} \cdot \boldsymbol{\psi}_{h}\right]\right]\right\|_{K}^{2}+\sum_{K} \alpha_{u}\left\|P_{u}^{\perp}\left[\lambda^{*} \nabla p_{h}\right]\right\|_{K}^{2} \\
& +\sum_{K} \alpha_{u}\left\|P_{u}^{\perp}\left[\lambda^{*} \rho \boldsymbol{a} \cdot \nabla \boldsymbol{u}_{h}\right]\right\|_{K}^{2}+\sum_{K} \alpha_{p}\left\|P_{p}^{\perp}\left[\lambda^{*} \nabla \cdot \boldsymbol{u}_{h}\right]\right\|_{K}^{2} \\
& +\sum_{K} \alpha_{\psi}\left(\frac{\lambda}{2 \lambda_{0}}\right)^{2}\left\|P_{\psi}^{\perp}\left[\frac{\lambda}{2 \lambda_{0}}\left(\boldsymbol{a} \cdot \nabla P_{\psi}\left[\boldsymbol{E} \cdot \boldsymbol{\psi}_{h}\right]-\dot{\boldsymbol{\psi}}_{h}^{* *}\right)\right]\right\|_{K}^{2}
\end{aligned}
$$

Even if $\alpha_{u}=\alpha_{p}=\alpha_{\psi}=0$, this estimate yields some stability provided $\lambda$ is small enough. In fact, this would be the estimate for the Galerkin method, which is the same as for the continuous problem. For the latter it would be possible to obtain pressure stability and stability for the velocity gradient through the use of appropriate inf-sup conditions. In the discrete case, we will not use these, but we will see how the stabilization terms allow us to prove the theorem.

Let us introduce $\boldsymbol{v}_{1} \equiv P_{u, 0}\left(\rho \boldsymbol{a} \cdot \nabla \boldsymbol{u}_{h}+\nabla p_{h}-\frac{\eta_{p}}{\lambda_{0}} \nabla \cdot P_{\psi}\left[\boldsymbol{E} \cdot \boldsymbol{\psi}_{h}\right]\right)$ and consider $\boldsymbol{V}_{h 1}=\alpha_{u} \lambda^{*}\left[\boldsymbol{v}_{1}, 0, \mathbf{0}\right]$. Taking this test function, using Schwarz's and Young's inequalities and the inverse estimate (4.1) we get

$$
\begin{aligned}
B_{\text {stab }}\left(\boldsymbol{U}_{h}, \boldsymbol{V}_{h 1}\right) & =B_{\psi}\left(\boldsymbol{U}_{h}, \boldsymbol{V}_{h 1}\right)+B^{*}\left(\boldsymbol{U}_{h}, \boldsymbol{V}_{h 1}\right) \\
& =B^{*}\left(\boldsymbol{U}_{h}, \boldsymbol{V}_{h 1}\right)+\sum_{K} \alpha_{u} \lambda^{*}\left\langle\frac{\eta_{p}}{\lambda_{0}} P_{\psi}\left[\boldsymbol{E} \cdot \boldsymbol{\psi}_{h}\right], \nabla^{\mathrm{s}} \boldsymbol{v}_{1}\right\rangle_{K}+\sum_{K} \alpha_{u} \lambda^{*}\left\langle\rho \boldsymbol{a} \cdot \nabla \boldsymbol{u}_{h}, \boldsymbol{v}_{1}\right\rangle_{K}
\end{aligned}
$$




$$
-\sum_{K} \alpha_{u} \lambda^{*}\left\langle p_{h}, \nabla \cdot \boldsymbol{v}_{1}\right\rangle_{K}-\sum_{K} \alpha_{u} \lambda^{*}\left[\frac{\varepsilon_{1}}{2}\left\|\boldsymbol{v}_{1}\right\|_{K}^{2}+\frac{1}{2 \varepsilon_{1}}\left(2 \eta_{e}\right)^{2} \frac{c_{\mathrm{inv}}^{2}}{h^{2}}\left\|\nabla^{\mathrm{s}} \boldsymbol{u}_{h}\right\|_{K}^{2}\right] .
$$

Integrating by parts the second and fourth terms and using the continuity assumed for the interpolation and the advection velocity, we get

$$
B_{\text {stab }}\left(\boldsymbol{U}_{h}, \boldsymbol{V}_{h 1}\right) \geq B^{*}\left(\boldsymbol{U}_{h}, \boldsymbol{V}_{h 1}\right)+\left(1-\frac{\varepsilon_{1}}{2}\right) \sum_{K} \alpha_{u} \lambda^{*}\left\|\boldsymbol{v}_{1}\right\|_{K}^{2}-\frac{1}{2 \varepsilon_{1}}\left(2 \eta_{e}\right)^{2} \frac{c_{\mathrm{inv}}^{2}}{h^{2}} \sum_{K} \alpha_{u} \lambda^{*}\left\|\nabla^{\mathrm{s}} \boldsymbol{u}_{h}\right\|_{K}^{2} .
$$

Repeated application of Schwarz's, Young's and the inequality $\|a+b+c\|^{2} \leq 4\|a\|^{2}+4\|b\|^{2}+2\|c\|^{2}$ and the inverse estimate (4.1) allow us to bound the stabilizing terms, and obtain:

$$
\begin{aligned}
B_{\mathrm{stab}}\left(\boldsymbol{U}_{h}, \boldsymbol{V}_{h 1}\right) \geq & \lambda^{*} \sum_{K} \alpha_{u} C_{u}\left\|\boldsymbol{v}_{1}\right\|_{K}^{2}-\lambda^{*} \sum_{K} \alpha_{u}\left(\frac{1}{\varepsilon_{1}} 2 \eta_{e}^{2} \alpha_{u} \frac{c_{\mathrm{inv}}^{2}}{h^{2}}+\frac{1}{\varepsilon_{4}} \alpha_{\psi}\left(\lambda^{*}\right)^{2}\right)\left\|\nabla^{\mathrm{s}} \boldsymbol{u}_{h}\right\|_{K}^{2} \\
& -\frac{1}{2 \varepsilon_{2}} \lambda^{*} \sum_{K} \alpha_{u}^{2}\|\boldsymbol{a}\|_{L^{\infty}(K)} \frac{\rho}{h}\left\|P_{u}^{\perp}\left[\rho \boldsymbol{a} \cdot \nabla \boldsymbol{u}_{h}\right]\right\|_{K}^{2}-\frac{1}{2 \varepsilon_{3}} \lambda^{*} \sum_{K} \alpha_{p}\left\|P_{p}^{\perp}\left[\nabla \cdot \boldsymbol{u}_{h}\right]\right\|_{K}^{2} \\
& -4 \frac{1}{2 \varepsilon_{4}} \lambda^{*} \sum_{K} \alpha_{\psi}\left\|P_{\psi}^{\perp}\left[\frac{\lambda}{2 \lambda_{0}}\left(\boldsymbol{a} \cdot \nabla P_{\psi}\left[\boldsymbol{E} \cdot \boldsymbol{\psi}_{h}\right]-\boldsymbol{\psi}_{h}^{* *}\right)\right]\right\|_{K}^{2} \\
& -4 \frac{1}{2 \varepsilon_{4}} \lambda^{*} \sum_{K} \alpha_{\psi}\left(\frac{\lambda}{2 \lambda_{0}}\right)^{2} 4\left\|\nabla^{\mathrm{s}} \boldsymbol{a}\right\|_{L^{\infty}(K)}^{2}\left\|P_{\psi}\left[\boldsymbol{E} \cdot \boldsymbol{\psi}_{h}\right]\right\|_{K}^{2},
\end{aligned}
$$

where

$$
C_{u}:=1-\frac{\varepsilon_{1}}{2}-c_{\mathrm{inv}}^{2} \alpha_{u}\left[\frac{\varepsilon_{2}}{2}\|\boldsymbol{a}\|_{L^{\infty}(K)} \frac{\rho}{h}+\frac{\varepsilon_{3}}{2} \frac{\alpha_{p}}{h^{2}}+\frac{\varepsilon_{4}}{2} \frac{\alpha_{\psi} \alpha_{u}}{h^{2}}\right],
$$

and $\varepsilon_{i}, i=2,3,4$ come again from different instances of the application of Young's inequality.

Let us consider now the test function $\boldsymbol{V}_{h 2}=\alpha_{p} \lambda^{*}\left[\mathbf{0}, q_{2}, \mathbf{0}\right]$, with $q_{2} \equiv P_{p}\left[\nabla \cdot \boldsymbol{u}_{h}\right]$. Using the same tools as above we get

$$
B_{\text {stab }}\left(\boldsymbol{U}_{h}, \boldsymbol{V}_{h 2}\right) \geq \lambda^{*} \sum_{K} \alpha_{p} C_{p}\left\|P_{p}\left[\nabla \cdot \boldsymbol{u}_{h}\right]\right\|_{K}^{2}-\lambda^{*} \sum_{K} \alpha_{u} \frac{\varepsilon_{5}}{2}\left\|P_{u}^{\perp}\left[\nabla p_{h}\right]\right\|_{K}^{2}
$$

where

$$
C_{p}:=1-\frac{1}{2 \varepsilon_{5}} \alpha_{p} \alpha_{u} \frac{c_{\mathrm{inv}}^{2}}{h^{2}}
$$

The next step is to consider the test function $\boldsymbol{V}_{h 3}=\alpha_{\psi}\left[\mathbf{0}, 0, \boldsymbol{\psi}_{3}\right]$, with

$$
\boldsymbol{\psi}_{3} \equiv P_{\psi}\left(\lambda^{*} \nabla^{\mathrm{s}} \boldsymbol{u}_{h}+\frac{\lambda}{2 \lambda_{0}}\left(\boldsymbol{a} \cdot \nabla P_{\psi}\left[\boldsymbol{E} \cdot \boldsymbol{\psi}_{h}\right]-\dot{\boldsymbol{\psi}}_{h}^{*}-\dot{\boldsymbol{\psi}}_{h}^{* *}\right)\right) .
$$

The process of bounding $B_{\text {stab }}\left(\boldsymbol{U}_{h}, \boldsymbol{V}_{h 3}\right)$ is similar to that of bounding $B_{\text {stab }}\left(\boldsymbol{U}_{h}, \boldsymbol{V}_{h 1}\right)$. Again, one has to apply repeatedly the same inequalities as before. Most details will be omitted.

Bounding first the Galerkin terms one gets:

$$
\begin{aligned}
B_{\text {stab }}\left(\boldsymbol{U}_{h}, \boldsymbol{V}_{h 3}\right) \geq & B^{*}\left(\boldsymbol{U}_{h}, \boldsymbol{V}_{h 3}\right)-\frac{1}{2 \lambda_{0}^{2}} \sum_{K} \frac{1}{2 \varepsilon_{6}}\left\|P_{\psi}\left[\boldsymbol{E} \cdot \boldsymbol{\psi}_{h}\right]\right\|_{K}^{2}-\frac{1}{2 \lambda_{0}^{2}} \sum \frac{\varepsilon_{6}}{2} \alpha_{\psi}\left\|\boldsymbol{\psi}_{3}\right\|_{K}^{2} \\
& +\sum_{K} \alpha_{\psi}\left(1-\frac{1}{\varepsilon_{7}}-\frac{1}{\varepsilon_{8}}\right)\left\|P_{\psi}\left[\lambda^{*} \nabla^{\mathrm{s}} \boldsymbol{u}_{h}\right]\right\|_{K}^{2}
\end{aligned}
$$




$$
\begin{aligned}
& +\sum_{K} \alpha_{\psi}\left(1-\varepsilon_{8}-\varepsilon_{9}\right)\left\|P_{\psi}\left[\frac{\lambda}{2 \lambda_{0}} \dot{\boldsymbol{\psi}}_{h}^{*}\right]\right\|_{K}^{2} \\
& +\sum_{K} \alpha_{\psi}\left(1-\varepsilon_{7}-\frac{1}{\varepsilon_{9}}\right)\left\|P_{\psi}\left[\frac{\lambda}{2 \lambda_{0}}\left(\boldsymbol{a} \cdot \nabla P_{\psi}\left[\boldsymbol{E} \cdot \boldsymbol{\psi}_{h}\right]-\dot{\boldsymbol{\psi}}_{h}^{* *}\right)\right]\right\|_{K}^{2},
\end{aligned}
$$

whereas for the stabilization terms one can get

$$
\begin{aligned}
B^{*}\left(\boldsymbol{U}_{h}, \boldsymbol{V}_{h 3}\right) \geq & -\sum_{K} \alpha_{u}\left(\frac{\eta_{p}}{\lambda_{0}}\right)^{2}\left[\frac{1}{2 \varepsilon_{10}}\left\|P_{u}^{\perp}\left[\nabla \cdot P_{\psi}\left[\boldsymbol{E} \cdot \boldsymbol{\psi}_{h}\right]\right]\right\|_{K}^{2}\right]-2 \frac{1}{2 \varepsilon_{11}} \sum_{K} \alpha_{\psi}\left\|P_{\psi}^{\perp}\left[\lambda^{*} \nabla^{\mathrm{s}} \boldsymbol{u}_{h}\right]\right\|_{K}^{2} \\
& -4 \frac{1}{2 \varepsilon_{11}} \sum_{K} \alpha_{\psi}\left\|P_{\psi}^{\perp}\left[\frac{\lambda}{2 \lambda_{0}}\left(\boldsymbol{a} \cdot \nabla P_{\psi}\left[\boldsymbol{E} \cdot \boldsymbol{\psi}_{h}\right]-\dot{\boldsymbol{\psi}}_{h}^{* *}\right)\right]\right\|_{K}^{2}-4 \frac{1}{2 \varepsilon_{11}} \sum_{K} \alpha_{\psi}\left\|P_{\psi}^{\perp}\left[\frac{\lambda}{2 \lambda_{0}} \dot{\boldsymbol{\psi}}_{h}^{*}\right]\right\|_{K}^{2} \\
& -\sum_{K} \alpha_{\psi}\left[\frac{\varepsilon_{10}}{2} \frac{c_{\mathrm{inv}}^{2}}{h^{2}} \alpha_{\psi} \alpha_{u}+\frac{\varepsilon_{11}}{2}\left\{4 \alpha_{\psi}^{2}\left(\frac{\lambda}{2 \eta_{p}} \frac{\|\boldsymbol{a}\|_{L^{\infty}(K)}}{h}\right)^{2}\right.\right. \\
& \left.\left.+4 \alpha_{\psi}^{2}\left(\frac{\lambda}{2 \eta_{p}}\left\|\nabla^{\mathrm{s}} \boldsymbol{a}\right\|_{L^{\infty}(K)}\right)^{2}+2 \alpha_{\psi}^{2}\left(\frac{\lambda}{2 \eta_{p}}\left\|\nabla^{\mathrm{as}} \boldsymbol{a}\right\|_{L^{\infty}(K)}\right)^{2}\right\}\right]\left\|\boldsymbol{\psi}_{3}\right\|_{K}^{2}
\end{aligned}
$$

Let us introduce the constant

$$
\begin{aligned}
C_{\psi}:= & \frac{1}{2 \eta_{p}} \frac{\varepsilon_{6}}{2} \alpha_{\psi}+\frac{\varepsilon_{10}}{2} \frac{c_{\text {inv }}^{2}}{h^{2}} \alpha_{\psi} \alpha_{u} \\
& +\frac{\varepsilon_{11}}{2}\left\{4 \alpha_{\psi}^{2}\left(\frac{\lambda}{2 \eta_{p}} \frac{\|\boldsymbol{a}\|_{L^{\infty}(K)}}{h}\right)^{2}+4 \alpha_{\psi}^{2}\left(\frac{\lambda}{2 \eta_{p}}\left\|\nabla^{\mathrm{s}} \boldsymbol{a}\right\|_{L^{\infty}(K)}\right)^{2}+2 \alpha_{\psi}^{2}\left(\frac{\lambda}{2 \eta_{p}}\left\|\nabla^{\mathrm{as}} \boldsymbol{a}\right\|_{L^{\infty}(K)}\right)^{2}\right\}
\end{aligned}
$$

and consider the inequality

$$
\left\|\boldsymbol{\psi}_{3}\right\|_{K}^{2} \geq-2\left\|P_{\psi}\left[\lambda^{*} \nabla^{\mathrm{s}} \boldsymbol{u}_{h}\right]\right\|^{2}-4\left\|P_{\psi}\left[\frac{\lambda}{2 \lambda_{0}}\left(\boldsymbol{a} \cdot \nabla P_{\psi}\left[\boldsymbol{E} \cdot \boldsymbol{\psi}_{h}\right]-\dot{\boldsymbol{\psi}}_{h}^{* *}\right)\right]\right\|^{2}-4\left\|P_{\psi}\left[\frac{\lambda}{2 \lambda_{0}} \dot{\boldsymbol{\psi}}_{h}^{*}\right]\right\|^{2}
$$

Now using (4.10) in (4.9) we obtain:

$$
\begin{aligned}
B_{\text {stab }}\left(\boldsymbol{U}_{h}, \boldsymbol{V}_{h 3}\right) \geq & -\frac{1}{2 \lambda_{0}} \sum_{K} \alpha_{\psi} \frac{1}{2 \varepsilon_{6}}\left\|P_{\psi}\left[\boldsymbol{E} \cdot \boldsymbol{\psi}_{h}\right]\right\|_{K}^{2}+\sum_{K} \alpha_{\psi}\left(1-\frac{1}{\varepsilon_{7}}-\frac{1}{\varepsilon_{8}}-2 C_{\psi}\right)\left\|P_{\psi}\left[\lambda^{*} \nabla^{\mathrm{s}} \boldsymbol{u}_{h}\right]\right\|_{K}^{2} \\
& +\sum_{K} \alpha_{\psi}\left(1-\varepsilon_{8}-\varepsilon_{9}-4 C_{\psi}\right)\left\|P_{\psi}\left[\frac{\lambda}{2 \lambda_{0}} \dot{\boldsymbol{\psi}}_{h}^{*}\right]\right\|_{K}^{2} \\
& +\sum_{K} \alpha_{\psi}\left(1-\varepsilon_{7}-\frac{1}{\varepsilon_{9}}-4 C_{\psi}\right)\left\|P_{\psi}\left[\frac{\lambda}{2 \lambda_{0}}\left(\boldsymbol{a} \cdot \nabla P_{\psi}\left[\boldsymbol{E} \cdot \boldsymbol{\psi}_{h}\right]-\dot{\boldsymbol{\psi}}_{h}^{* *}\right)\right]\right\|_{K}^{2} \\
& -\frac{1}{2 \varepsilon_{10}} \sum_{K} \alpha_{u}\left(\frac{\eta_{p}}{\lambda_{0}}\right)^{2}\left\|P_{u}^{\perp}\left[\nabla \cdot P_{\psi}\left[\boldsymbol{E} \cdot \boldsymbol{\psi}_{h}\right]\right]\right\|_{K}^{2}-2 \frac{1}{2 \varepsilon_{11}} \sum_{K} \alpha_{\psi}\left\|P_{\psi}^{\perp}\left[\lambda^{*} \nabla^{\mathrm{s}} \boldsymbol{u}_{h}\right]\right\|_{K}^{2} \\
& -4 \frac{1}{2 \varepsilon_{11}} \sum_{K} \alpha_{\psi}\left\|P_{\psi}^{\perp}\left[\frac{\lambda}{2 \lambda_{0}}\left(\boldsymbol{a} \cdot \nabla P_{\psi}\left[\boldsymbol{E} \cdot \boldsymbol{\psi}_{h}\right]-\dot{\boldsymbol{\psi}}_{h}^{* *}\right)\right]\right\|_{K}^{2} \\
& -4 \frac{1}{2 \varepsilon_{11}}\left(\frac{\lambda}{2 \lambda_{0}}\right)^{2} \sum_{K} \alpha_{\psi} 4\left\|\nabla^{\mathrm{s}} \boldsymbol{a}\right\|_{L^{\infty}(K)}^{2}\left\|P_{\psi}\left[\boldsymbol{E} \cdot \boldsymbol{\psi}_{h}\right]\right\|_{K}^{2} \cdot
\end{aligned}
$$


It can be checked that the constants $\varepsilon_{i}, i=1, \ldots, 11$ arising from Young's inequality can be taken such that

$$
C_{u}>0, \quad C_{p}>0, \quad C_{\psi}>0,
$$

where $C_{u}, C_{p}$ and $C_{\psi}$ are given in (4.6), (4.8) and (4.11), respectively.

Lastly, let us consider $\boldsymbol{V}_{h}=\boldsymbol{U}_{h 1}+\theta_{1} \boldsymbol{V}_{h 1}+\theta_{2} \boldsymbol{V}_{h 2}+\theta_{3} \boldsymbol{V}_{h 3}$. The parameters $\theta_{i}$ can be chosen small enough so as to obtain, from (4.5), (4.7) and (4.12):

$$
\begin{aligned}
B_{\mathrm{stab}}\left(\boldsymbol{U}_{h}, \boldsymbol{V}_{h}\right) \geq & 2 \eta_{e} \sum_{K} C_{1}\left\|\nabla^{\mathrm{s}} \boldsymbol{u}_{h}\right\|_{K}^{2}+\frac{\eta_{p}}{\lambda_{0}^{2}} \sum_{K} C_{2}\left\|P_{\psi}\left[\boldsymbol{E} \cdot \boldsymbol{\psi}_{h}\right]\right\|_{K}^{2} \\
& +\sum_{K} \alpha_{u} C_{3}\left\|P_{u, 0}\left[\rho \boldsymbol{a} \cdot \nabla \boldsymbol{u}_{h}+P_{\psi}-\frac{\eta_{p}}{\lambda_{0}} \nabla \cdot P_{\psi}\left[\boldsymbol{E} \cdot \boldsymbol{\psi}_{h}\right]\right]\right\|_{K}^{2} \\
& +\sum_{K} \alpha_{u} C_{4}\left\|P_{u}^{\perp}\left[\frac{\eta_{p}}{\lambda_{0}} \nabla \cdot P_{\psi}\left[\boldsymbol{E} \cdot \boldsymbol{\psi}_{h}\right]\right]\right\|_{K}^{2}+\sum_{K} \alpha_{u} C_{5}\left\|P_{u}^{\perp}\left[\nabla p_{h}\right]\right\|_{K}^{2} \\
& +\sum_{K} \alpha_{u} C_{6}\left\|P_{u}^{\perp}\left[\rho \boldsymbol{a} \cdot \nabla \boldsymbol{u}_{h}\right]\right\| \\
& +\sum_{K} \alpha_{p} C_{7}\left\|P_{p}^{\perp}\left[\nabla \cdot \boldsymbol{u}_{h}\right]\right\|_{K}^{2}+\sum_{K} \alpha_{p} C_{8}\left\|P_{p}\left[\nabla \cdot \boldsymbol{u}_{h}\right]\right\|^{2} \\
& +\sum_{K} \alpha_{\psi} C_{9}\left\|P_{\psi}^{\perp}\left[\frac{\lambda}{2 \lambda_{0}}\left(\boldsymbol{a} \cdot \nabla P_{\psi}\left[\boldsymbol{E} \cdot \boldsymbol{\psi}_{h}\right]-\dot{\boldsymbol{\psi}}_{h}^{* *}\right)\right]\right\|_{K}^{2} \\
& +\sum_{K} \alpha_{\psi} C_{10}\left\|P_{\psi}\left[\frac{\lambda}{2 \lambda_{0}}\left(\boldsymbol{a} \cdot \nabla P_{\psi}\left[\boldsymbol{E} \cdot \boldsymbol{\psi}_{h}\right]-\dot{\boldsymbol{\psi}}_{h}^{* *}\right)\right]\right\|_{K}^{2} \\
& +\sum_{K} \alpha_{\psi} C_{11}\left\|P_{\psi}\left[\nabla^{\mathrm{s}} \boldsymbol{u}_{h}\right]\right\|_{K}^{2}+\sum_{K} \alpha_{\psi} C_{12}\left\|P_{\psi}\left[\frac{\lambda}{2 \lambda_{0}} \dot{\boldsymbol{\psi}}_{h}^{*}\right]\right\|_{K}^{2}
\end{aligned}
$$

with the various constants appearing in this expression given by

$$
\begin{aligned}
C_{1}= & \lambda^{*}-\frac{\eta_{p} \varepsilon_{0} \lambda^{*}}{2 \eta_{e}}-\frac{\left(\lambda^{*}\right)^{2} \alpha_{\psi}}{2 \eta_{e}}-\frac{\theta_{1} \lambda^{*} \alpha_{u}}{2 \eta_{e}}\left(\frac{1}{2 \varepsilon_{1}}\left(2 \eta_{e}\right)^{2} \alpha_{u} \frac{c_{\mathrm{inv}}^{2}}{h^{2}}+2 \frac{1}{2 \varepsilon_{4}}\left(\lambda^{*}\right)^{2} \alpha_{\psi}\right)-\frac{\theta_{3} \alpha_{\psi}}{2 \eta_{e}} 2 \frac{\eta_{p}}{\lambda_{0}} \frac{1}{2 \varepsilon_{11}}\left(\lambda^{*}\right)^{2}, \\
C_{2}= & \left(1-\frac{\lambda^{*}}{\varepsilon_{0}}-\frac{\lambda}{\lambda_{0}}\|\nabla \boldsymbol{a}\|_{L^{\infty}(K)}\right)-\frac{\lambda_{0}^{2}}{\eta_{p}} \alpha_{\psi}\left(\frac{\lambda}{2 \lambda_{0}}\right)^{2} 4\left\|\nabla^{\mathrm{s}} \boldsymbol{a}\right\|_{L^{\infty}(K)}^{2}\left(1-\theta_{1} 4 \frac{1}{2 \varepsilon_{4}} \lambda^{*}\right) \\
& -\theta_{3} \frac{\lambda_{0}^{2}}{\eta_{p}}\left(\frac{1}{2 \lambda_{0}} \frac{1}{2 \varepsilon_{6}}+4 \frac{1}{2 \varepsilon_{11}}\left(\frac{\lambda}{2 \lambda_{0}}\right)^{2} \alpha_{\psi} 4\left\|\nabla^{\mathrm{s}} \boldsymbol{a}\right\|_{L^{\infty}(K)}^{2}\right), \\
C_{3}= & \lambda^{*} \theta_{1} C_{u}=\lambda^{*} \theta_{1}\left(1-\frac{\varepsilon_{1}}{2}-c_{\mathrm{inv}}^{2} \alpha_{u}\left[\frac{\varepsilon_{2}}{2}\|\boldsymbol{a}\|_{L^{\infty}(K)} \frac{\rho}{h}+\frac{\varepsilon_{3}}{2} \frac{\alpha_{p}}{h^{2}}+\frac{\varepsilon_{4}}{2} \frac{\alpha_{\psi}}{h^{2}} \alpha_{u}\right]\right), \\
C_{4}= & 1-\theta_{3} \frac{1}{2 \varepsilon_{10}} \\
C_{5}= & \lambda^{*}\left(1-\frac{\varepsilon_{5}}{2} \theta_{2}\right), \\
C_{6}= & \left(\lambda^{*}\right)^{2}\left(1-\lambda^{*} \theta_{1} \frac{1}{2 \varepsilon_{2}} \alpha_{u}\|\boldsymbol{a}\|_{L^{\infty}(K)} \frac{\rho}{h}\right), \\
C_{7}= & \lambda^{*}\left(\lambda^{*}-\theta_{1} \frac{1}{2 \varepsilon_{3}}\right),
\end{aligned}
$$




$$
\begin{aligned}
C_{8} & =\lambda^{*} \theta_{2} C_{p}=\lambda^{*} \theta_{2}\left(1-\frac{1}{2 \varepsilon_{5}} \alpha_{p} \alpha_{u} \frac{c_{\text {inv }}^{2}}{h^{2}}\right), \\
C_{9} & =1-4 \frac{1}{2 \varepsilon_{4}} \lambda^{*}-\theta_{3} 4 \frac{1}{2 \varepsilon_{11}} \\
C_{10} & =\theta_{3}\left(1-\varepsilon_{7}-\frac{1}{\varepsilon_{9}}-4 C_{\psi}\right), \\
C_{11} & =\theta_{3}\left(1-\frac{1}{\varepsilon_{7}}-\frac{1}{\varepsilon_{8}}-2 C_{\psi}\right), \\
C_{12} & =\theta_{3}\left(1-\varepsilon_{8}-\varepsilon_{9}-4 C_{\psi}\right),
\end{aligned}
$$

all positive for $\lambda$ small enough and the constants $c_{i}$ of the stability parameters large enough, and bounded by virtue of Assumption H2. Note that these constants will be larger for smaller values of $\lambda$, and the constant $C$ in the inf-sup condition stated by the theorem will also be larger. There is a tradeoff between the smallness of $\lambda$ and that of $C$ when the velocity or its gradients are large (or viscosities are small).

Comparing the terms in the right-hand-side of (4.13) and the definition (4.2), it is seen that the former bounds $\left\|P_{u, 0}\left[\rho \boldsymbol{a} \cdot \nabla \boldsymbol{u}_{h}+\nabla p_{h}-\frac{\eta_{p}}{\lambda_{0}} \nabla \cdot P_{\psi}\left[\boldsymbol{E} \cdot \boldsymbol{\psi}_{h}\right]\right]\right\|$ and $\left\|P_{u}^{\perp}\left[\rho \boldsymbol{a} \cdot \nabla \boldsymbol{u}_{h}+\nabla p_{h}-\frac{\eta_{p}}{\lambda_{0}} \nabla \cdot P_{\psi}\left[\boldsymbol{E} \cdot \boldsymbol{\psi}_{h}\right]\right]\right\|$. Assumption H2 allows us to guarantee that it also bounds $\left\|\rho \boldsymbol{a} \cdot \nabla \boldsymbol{u}_{h}+\nabla p_{h}-\frac{\eta_{p}}{\lambda_{0}} \nabla \cdot P_{\psi}\left[\boldsymbol{E} \cdot \boldsymbol{\psi}_{h}\right]\right\|$. Therefore, for each $\boldsymbol{U}_{h}$ we have found $\boldsymbol{V}_{h}$ such that

$$
B_{\text {stab }}\left(\boldsymbol{U}_{h}, \boldsymbol{V}_{h}\right) \geq C\left\|\boldsymbol{U}_{h}\right\|_{W}^{2} .
$$

In fact, it is seen from (4.13) that we could have included term $\alpha_{\psi} C_{12}\left\|P_{\psi}\left[\frac{\lambda}{2 \lambda_{0}} \dot{\boldsymbol{\psi}}_{h}^{*}\right]\right\|^{2}$ in the working norm, which gives control on the FE part of $P_{\psi}\left[\boldsymbol{E} \cdot \boldsymbol{\psi}_{h}\right] \cdot \nabla^{\mathrm{s}} \boldsymbol{a}+\nabla^{\mathrm{s}} \boldsymbol{a} \cdot P_{\psi}\left[\boldsymbol{E} \cdot \boldsymbol{\psi}_{h}\right]$. On the other hand, it is easily checked that $\left\|\boldsymbol{V}_{h}\right\|_{W} \leq C\left\|\boldsymbol{U}_{h}\right\|_{W}$; we will omit the immediate proof. Using this fact we have shown that for each $\boldsymbol{U}_{h} \in \mathcal{X}_{h}$ there exist $\boldsymbol{V}_{h} \in \mathcal{X}_{h}$ such that

$$
B_{\text {stab }}\left(\boldsymbol{U}_{h}, \boldsymbol{V}_{h}\right) \geq C\left\|\boldsymbol{U}_{h}\right\|_{W}\left\|\boldsymbol{V}_{h}\right\|_{W},
$$

from where theorem follows.

Now, we will define the error function of the method. Let us consider a FE space $\mathcal{W}_{h}$, made of piecewise continuous polynomial functions of degree $k_{v}$. Given a function $v \in H^{k_{v}^{\prime}+1}(\Omega)$, for $i=0,1$ the interpolation errors $\varepsilon_{i}(v)$ are defined as

$$
\inf _{v_{h} \in \mathcal{W}_{h}} \sum_{K}\left\|v-v_{h}\right\|_{H^{i}(K)} \leq C h^{k_{v}^{\prime \prime}+1-i} \sum_{K}\|v\|_{H^{k_{v}^{\prime \prime}+1}(K)}=: \sum_{K} \varepsilon_{i, K}(v)=: \varepsilon_{i}(v),
$$

where $k_{v}^{\prime \prime}=\min \left(k_{v}, k_{v}^{\prime}\right)$. We will denote from this point by $\tilde{v}_{h}$ the best approximation of $v$ in $\mathcal{W}_{h}$. Note that $\varepsilon_{0}(v)=h \varepsilon_{1}(v)$. In the case of $v=\boldsymbol{\psi}$, it is understood that $\varepsilon_{i}(\boldsymbol{\psi}):=\inf _{\boldsymbol{\psi}_{h} \in \boldsymbol{\Upsilon}_{h}} \sum_{K}\left\|\boldsymbol{E} \cdot \boldsymbol{\psi}-\boldsymbol{E} \cdot \boldsymbol{\psi}_{h}\right\|_{H^{i}(K)}$.

The objective of what follows is to show that the error function of the method we propose is:

$$
\mathcal{E}(h):=\sqrt{\eta_{0}} \varepsilon_{1}(\boldsymbol{u})+\sqrt{\eta_{0}} \sum_{K} \sqrt{\operatorname{Re}_{K}} \varepsilon_{1, K}(\boldsymbol{u})+\frac{\sqrt{\eta_{0}}}{\lambda_{0}} \varepsilon_{0}(\boldsymbol{\psi})+\frac{\sqrt{\eta_{0}}}{\lambda_{0}} \sum_{K} \sqrt{\mathrm{We}_{K}} \varepsilon_{0, K}(\boldsymbol{\psi})+\frac{1}{\sqrt{\eta_{0}}} \varepsilon_{0}(p),
$$

where

$$
\operatorname{Re}_{K}:=\frac{\rho\|\boldsymbol{a}\|_{L^{\infty}(K)} h}{\eta_{0}}, \quad \mathrm{We}_{K}:=\frac{\lambda\|\boldsymbol{a}\|_{L^{\infty}(K)}}{h}
$$

are the element (or cell) Reynolds and Weissenberg numbers, respectively.

At this point, a very important remark is needed. In [6] it is proved that the FE method proposed for the standard formulation of the viscoelastic flow problem is stable and has an error function similar to (4.14) but 
with a major difference: now the term that accounts for the error of the logarithm of the conformation tensor has a factor $\lambda_{0}^{-1}$ in front. This is a very important improvement, as the growth of the error with the elasticity of the flow will be significantly reduced in the log-conformation formulation with respect to the standard one. Nevertheless, there is a remark to be made, both for the standard and the log-conformation approaches: we will show that (4.14) is the error function of the method up to a certain $\mathrm{We}_{K}$ that needs to be sufficiently small and that is unknown in both cases. Obviously, the growth of the error with the element Reynolds number is the same.

Lemma 4.2 (Consistency). Let $\boldsymbol{U} \in \mathcal{X}$ be the solution of the continuous problem and $\boldsymbol{U}_{h} \in \mathcal{X}_{h}$ the $F E$ solution. If $\boldsymbol{f} \in \mathcal{V}_{h}$ and $\boldsymbol{U}$ is regular enough, so that $B_{\text {stab }}\left(\boldsymbol{U}, \boldsymbol{V}_{h}\right)$ is well defined, then

$$
B_{\text {stab }}\left(\boldsymbol{U}-\boldsymbol{U}_{h}, \boldsymbol{V}_{h}\right) \leq C \mathcal{E}(h)\left\|\boldsymbol{V}_{h}\right\|_{W},
$$

for all $\boldsymbol{V}_{h} \in \mathcal{X}_{h}$, where $\mathcal{E}(h)$ is defined by (4.14) and the constant $C$ is independent of the physical properties.

Proof. Galerkin terms do not contribute to the consistency error. In addition, the contribution of the constitutive and the continuity equations in the stabilization terms are residual based, therefore the consistency is satisfied by construction. Therefore we only have to show as $S_{1}^{\perp}$ has consistency error bounded as the lemma indicates. This is proved from the fact that the orthogonal projection $P^{\perp}$ onto an appropriate FE space satisfies $\left\|P^{\perp}(v)\right\| \leq C \varepsilon_{0}(v)$ for any function $v$. Details are omitted.

To show that the interpolation error is also $\mathcal{E}(h)$ we require a technical assumption that states that for any element $\chi_{h} \in \Upsilon_{h}, \boldsymbol{E} \cdot \boldsymbol{\chi}_{h}$ is close to a finite element function and its derivatives are close to the derivatives of a finite element function. Note that if $\chi_{h}$ is a stress, we may scale it by $\frac{\lambda_{0}}{\eta_{p}}$ to make it dimensionless. The condition we need may be expressed as follows:

Assumption H3 For all $\boldsymbol{\chi}_{h} \in \boldsymbol{\Upsilon}_{h}$ if $\mathcal{M}$ is a bounded linear operator of $\boldsymbol{\chi}_{h}$ and $\nabla \boldsymbol{\chi}_{h}$, there holds

$$
\left\|\mathcal{M}\left(\boldsymbol{\chi}_{h}, \nabla \chi_{h}\right)\right\|_{K} \leq C\left\|\mathcal{M}\left(P_{\psi}\left[\boldsymbol{E} \cdot \boldsymbol{\chi}_{h}\right], \nabla P_{\psi}\left[\boldsymbol{E} \cdot \boldsymbol{\chi}_{h}\right]\right)\right\|_{K}, \quad K \in \mathcal{T}_{h}
$$

Lemma 4.3 (Interpolation error). Let $\boldsymbol{U} \in \mathcal{X}$ be the solution of the continuous problem, assumed to be regular enough, and $\tilde{\boldsymbol{U}}_{h} \in \mathcal{X}_{h}$ its best FE approximation. Then, under Assumption H3, the following estimates hold:

$$
\begin{aligned}
B_{\text {stab }}\left(\boldsymbol{U}-\tilde{\boldsymbol{U}}_{h}, \boldsymbol{V}_{h}\right) & \leq C \mathcal{E}(h)\left\|\boldsymbol{V}_{h}\right\|_{W}, \\
\left\|\boldsymbol{U}-\tilde{\boldsymbol{U}}_{h}\right\|_{W} & \leq C \mathcal{E}(h) .
\end{aligned}
$$

Proof. Set $\boldsymbol{e}_{u}=\boldsymbol{u}-\tilde{\boldsymbol{u}}_{h} ; \boldsymbol{e}_{p}=p-\tilde{p}_{h}$ and $\boldsymbol{e}_{\psi}=\boldsymbol{\psi}-\tilde{\boldsymbol{\psi}}_{h}$. Firstly we will prove inequality (4.17):

$$
\begin{aligned}
\left\|\boldsymbol{U}-\tilde{\boldsymbol{U}}_{h}\right\|_{W}^{2}= & 2 \eta_{e}\left\|\nabla^{\mathrm{s}} \boldsymbol{e}_{u}\right\|^{2}+\frac{\eta_{p}}{\lambda_{0}^{2}}\left\|P_{\psi}\left[\boldsymbol{E} \cdot \boldsymbol{e}_{\psi}\right]\right\|^{2}+\overbrace{\sum_{K} \alpha_{u}\left\|\rho \boldsymbol{a} \cdot \nabla \boldsymbol{e}_{u}+\nabla \boldsymbol{e}_{p}-\frac{\eta_{p}}{\lambda_{0}} \nabla \cdot P_{\psi}\left[\boldsymbol{E} \cdot \boldsymbol{e}_{\psi}\right]\right\|_{K}^{2}}^{(1)} \\
& +\underbrace{\sum_{K} \alpha_{u}\left\|P_{u}^{\perp}\left[\rho \boldsymbol{a} \cdot \nabla \boldsymbol{e}_{u}\right]\right\|_{K}^{2}}_{(2)}+\underbrace{\sum_{K} \alpha_{u}\left\|P_{u}^{\perp}\left[\nabla \boldsymbol{e}_{p}\right]\right\|_{K}^{2}}_{(3)}+\underbrace{\sum_{K} \alpha_{u}\left\|P_{u}^{\perp}\left[\frac{\eta_{p}}{\lambda_{0}} \nabla \cdot P_{\psi}\left[\boldsymbol{E} \cdot \boldsymbol{e}_{\psi}\right]\right]\right\|_{K}^{2}}_{(4)} \\
& +\underbrace{\sum_{K} \alpha_{p}\left\|\nabla \cdot \boldsymbol{e}_{u}\right\|_{K}^{2}}_{(5)}+\underbrace{\sum_{K} \alpha_{\psi} \| \frac{\lambda}{2 \lambda_{0}}\left(\boldsymbol{a} \cdot \nabla P_{\psi}\left[\boldsymbol{E} \cdot \boldsymbol{e}_{\psi}\right]\right.}_{(6)}-\dot{\boldsymbol{e}}_{\psi}^{* *}) \|_{K}^{2}
\end{aligned}
$$


where

$$
\begin{aligned}
(1) & \leq \sum_{K} \alpha_{u}\left(2\left\|\rho \boldsymbol{a} \cdot \nabla \boldsymbol{e}_{u}\right\|_{K}^{2}+2\left\|\nabla \boldsymbol{e}_{p}\right\|_{K}^{2}+\left\|\frac{\eta_{p}}{\lambda_{0}} \nabla \cdot P_{\psi}\left[\boldsymbol{E} \cdot \boldsymbol{e}_{\psi}\right]\right\|_{K}^{2}\right) \\
& \leq \sum_{K} \alpha_{u}\left(2 \rho^{2}\|\boldsymbol{a}\|_{L^{\infty}(K)}^{2} \varepsilon_{1}^{2}(\boldsymbol{u})+\frac{2}{h^{2}} \varepsilon_{0}^{2}(p)+\frac{1}{h^{2}} \frac{\eta_{p}^{2}}{\lambda_{0}^{2}} \varepsilon_{0}^{2}(\boldsymbol{\psi})\right) \\
(2) & \leq \sum_{K} \alpha_{u} \rho^{2}\|\boldsymbol{a}\|_{L^{\infty}(K)}^{2} \varepsilon_{1}^{2}(\boldsymbol{u}), \\
(3) & \leq \sum_{K} \alpha_{u} \frac{1}{h^{2}} \varepsilon_{0}^{2}(p), \\
(4) & \leq \sum_{K} \alpha_{u} \frac{1}{h^{2}} \frac{\eta_{p}^{2}}{\lambda_{0}^{2}} \varepsilon_{0}^{2}(\boldsymbol{\psi}), \\
(5) & \leq \sum_{K} \alpha_{p} \varepsilon_{1}^{2}(\boldsymbol{u}), \\
(6) & \leq \sum_{K} \alpha_{\psi}\left(\left(\frac{\lambda}{2 \lambda_{0}}\right)^{2}\|\boldsymbol{a}\|_{L^{\infty}(K)}^{2} \frac{1}{h^{2}} \varepsilon_{0}^{2}(\boldsymbol{\psi})+\left(\frac{\lambda}{2 \lambda_{0}}\right)^{2} 2\left\|\nabla^{\text {as }} \boldsymbol{a}\right\|_{L^{\infty}(K)}^{2} \varepsilon_{0}^{2}(\boldsymbol{\psi})\right) .
\end{aligned}
$$

Estimate (4.17) follows form the definitions of the error function and the expression of the stabilization parameters.

Now we will prove (4.16). Taking $\boldsymbol{V}_{h}=\left[\boldsymbol{v}_{h}, q_{h}, \boldsymbol{\chi}_{h}\right]$, we get:

$$
\begin{aligned}
B_{\text {stab }}\left(\boldsymbol{U}-\tilde{\boldsymbol{U}}_{h}, \boldsymbol{V}_{h}\right)= & \frac{\eta_{p}}{\lambda_{0}}\left(P_{\psi}\left[\boldsymbol{E} \cdot \boldsymbol{e}_{\psi}\right], \nabla^{\mathrm{s}} \boldsymbol{v}_{h}\right)+2 \eta_{e}\left(\nabla^{\mathrm{s}} \boldsymbol{e}_{u}, \nabla^{\mathrm{s}} \boldsymbol{v}_{h}\right)+\left\langle\rho \boldsymbol{a} \cdot \nabla \boldsymbol{e}_{u}, \boldsymbol{v}_{h}\right\rangle-\left(\boldsymbol{e}_{p}, \nabla \cdot \boldsymbol{v}_{h}\right) \\
& +\left(\nabla \cdot \boldsymbol{e}_{u}, q_{h}\right)+\frac{1}{2 \lambda_{0}}\left(P_{\psi}\left[\boldsymbol{E} \cdot \boldsymbol{e}_{\psi}\right], \boldsymbol{\chi}_{h}\right)-\left(\nabla^{\mathrm{s}} \boldsymbol{e}_{u}, \boldsymbol{\chi}_{h}\right) \\
& +\frac{\lambda}{2 \lambda_{0}}\left(\boldsymbol{a} \cdot \nabla P_{\psi}\left[\boldsymbol{E} \cdot \boldsymbol{e}_{\psi}\right]+2 \nabla^{\mathrm{s}} \boldsymbol{e}_{u}-P_{\psi}\left[\boldsymbol{E} \cdot \boldsymbol{e}_{\psi}\right] \cdot \nabla \boldsymbol{a}-(\nabla \boldsymbol{a})^{T} \cdot P_{\psi}\left[\boldsymbol{E} \cdot \boldsymbol{e}_{\psi}\right], \boldsymbol{\chi}_{h}\right) \\
& +\sum_{K} \alpha_{u}\left\langle P_{u}^{\perp}\left[-\frac{\eta_{p}}{\lambda_{0}} \nabla \cdot P_{\psi}\left[\boldsymbol{E} \cdot \boldsymbol{e}_{\psi}\right]\right],-\nabla \cdot \boldsymbol{\chi}_{h}\right\rangle_{K}+\sum_{K} \alpha_{u}\left\langle P_{u}^{\perp}\left[\nabla \boldsymbol{e}_{p}\right], \nabla q_{h}\right\rangle_{K} \\
& +\sum_{K} \alpha_{u}\left\langle P_{u}^{\perp}\left[\rho \boldsymbol{a} \cdot \nabla \boldsymbol{e}_{u}\right], \rho \boldsymbol{a} \cdot \nabla \boldsymbol{v}_{h}\right\rangle_{K}+\sum_{K} \alpha_{p}\left\langle P_{p}^{\perp}\left[\nabla \cdot \boldsymbol{e}_{u}\right], \nabla \cdot \boldsymbol{v}_{h}\right\rangle_{K} \\
& +\sum_{K} \alpha_{\psi}\left\langle P_{\psi}^{\perp}\left[\nabla^{\mathrm{s}} \boldsymbol{e}_{u}+\frac{\lambda}{2 \lambda_{0}}\left(\boldsymbol{a} \cdot \nabla P_{\psi}\left[\boldsymbol{E} \cdot \boldsymbol{e}_{\psi}\right]-P_{\psi}\left[\boldsymbol{E} \cdot \boldsymbol{e}_{\psi}\right] \cdot \nabla \boldsymbol{a}-(\nabla \boldsymbol{a})^{T} \cdot P_{\psi}\left[\boldsymbol{E} \cdot \boldsymbol{e}_{\psi}\right]\right)\right],\right. \\
& \left.-\nabla^{\mathrm{s}} \boldsymbol{v}_{h}+\frac{\lambda}{2 \eta_{p}}\left(\boldsymbol{a} \cdot \nabla \boldsymbol{\chi}_{h}+\boldsymbol{\chi}_{h} \cdot(\nabla \boldsymbol{a})^{T}+\nabla \boldsymbol{a} \cdot \boldsymbol{\chi}_{h}\right)\right\rangle_{K} .
\end{aligned}
$$

Using Schwarz's inequality, integrating by parts the convective term and the continuity equation and using the inverse estimate (4.1) and Assumption H3, we get:

$$
\begin{aligned}
B_{\text {stab }}\left(\boldsymbol{U}-\tilde{\boldsymbol{U}}_{h}, \boldsymbol{V}_{h}\right) \leq & \frac{\eta_{p}}{\lambda_{0}} \varepsilon_{0}(\boldsymbol{\psi})\left\|\nabla^{\mathrm{s}} \boldsymbol{v}_{h}\right\|+2 \eta_{e} \varepsilon_{1}(\boldsymbol{u})\left\|\nabla^{\mathrm{s}} \boldsymbol{v}_{h}\right\|+\varepsilon_{0}(p)\left\|\nabla \cdot \boldsymbol{v}_{h}\right\| \\
& +h \varepsilon_{1}(\boldsymbol{u})\left\|\rho \boldsymbol{a} \cdot \nabla \boldsymbol{v}_{h}+\nabla q_{h}-\nabla \cdot P_{\psi}\left[\boldsymbol{E} \cdot \boldsymbol{\chi}_{h}\right]\right\| \\
& +\left(\frac{1}{2 \lambda_{0}} \varepsilon_{0}(\boldsymbol{\psi})+\left(2+k^{-1}\right) \varepsilon_{1}(\boldsymbol{u})\right)\left\|P_{\psi}\left[\boldsymbol{E} \cdot \boldsymbol{\chi}_{h}\right]\right\| \\
& +\frac{\eta_{p}}{\lambda_{0}} \varepsilon_{0}(\boldsymbol{\psi}) \sum_{K}\left\|\frac{\lambda}{2 \eta_{p}}\left(\boldsymbol{a} \cdot \nabla P_{\psi}\left[\boldsymbol{E} \cdot \boldsymbol{\chi}_{h}\right]+P_{\psi}\left[\boldsymbol{E} \cdot \boldsymbol{\chi}_{h}\right] \cdot(\nabla \boldsymbol{a})^{T}+\nabla \boldsymbol{a} \cdot P_{\psi}\left[\boldsymbol{E} \cdot \boldsymbol{\chi}_{h}\right]\right)\right\|_{K}
\end{aligned}
$$




$$
\begin{aligned}
& +\sum_{K} \alpha_{u} \frac{\eta_{p}}{\lambda_{0}} \frac{1}{h} \varepsilon_{0}(\psi)\left\|P_{u}^{\perp}\left[\nabla \cdot P_{\psi}\left[\boldsymbol{E} \cdot \boldsymbol{\chi}_{h}\right]\right]\right\|_{K}+\sum_{K} \alpha_{u} \frac{1}{h} \varepsilon_{0}(p)\left\|P_{u}^{\perp}\left[\nabla q_{h}\right]\right\|_{K} \\
& +\sum_{K} \alpha_{u} \rho\|\boldsymbol{a}\|_{L^{\infty}(K)} \varepsilon_{1}(\boldsymbol{u})\left\|P_{u}^{\perp}\left[\rho \boldsymbol{a} \cdot \nabla \boldsymbol{v}_{h}\right]\right\|_{K}+\sum_{K} \alpha_{p} \varepsilon_{1}(\boldsymbol{u})\left\|\nabla \cdot \boldsymbol{v}_{h}\right\|_{K} \\
& +\sum_{K} \alpha_{\psi} \varepsilon_{1}(\boldsymbol{u})\left\|\nabla^{\mathrm{s}} \boldsymbol{v}_{h}\right\|_{K} \\
& +\sum_{K} \alpha_{\psi} \varepsilon_{1}(\boldsymbol{u})\left\|\frac{\lambda}{2 \eta_{p}}\left(\boldsymbol{a} \cdot \nabla P_{\psi}\left[\boldsymbol{E} \cdot \boldsymbol{\chi}_{h}\right]+P_{\psi}\left[\boldsymbol{E} \cdot \boldsymbol{\chi}_{h}\right] \cdot(\nabla \boldsymbol{a})^{T}+\nabla \boldsymbol{a} \cdot P_{\psi}\left[\boldsymbol{E} \cdot \boldsymbol{\chi}_{h}\right]\right)\right\|_{K} \\
& +\sum_{K} \alpha_{\psi} \varepsilon_{0}(\boldsymbol{\psi}) \frac{\lambda}{2 \lambda_{0}}\left(\frac{\|\boldsymbol{a}\|_{L^{\infty}(K)}}{h}+2\|\nabla \boldsymbol{a}\|_{L^{\infty}(K)}\right) \\
& \times\left(\left\|\nabla^{\mathrm{s}} \boldsymbol{v}_{h}\right\|_{K}+\left\|\frac{\lambda}{2 \eta_{p}}\left(\boldsymbol{a} \cdot \nabla P_{\psi}\left[\boldsymbol{E} \cdot \boldsymbol{\chi}_{h}\right]+P_{\psi}\left[\boldsymbol{E} \cdot \boldsymbol{\chi}_{h}\right] \cdot(\nabla \boldsymbol{a})^{T}+\nabla \boldsymbol{a} \cdot P_{\psi}\left[\boldsymbol{E} \cdot \boldsymbol{\chi}_{h}\right]\right)\right\|_{K}\right),
\end{aligned}
$$

where we have used that $\lambda_{0}=k \lambda$ (again, the case $\lambda$ small is easier). The results follows reorganizing terms, using the expressions of the stabilization parameters and of $\eta_{e}$ and $\eta_{p}$ in terms of $\eta_{0}$.

Theorem 4.4 (Convergence). Let $\boldsymbol{U}=[\boldsymbol{u}, p, \boldsymbol{\psi}] \in \mathcal{X}$ be the solution of the continuous problem, and suppose that the assumptions of Theorem 4.1 and Lemma 4.3 hold. Then there exist a constant $C>0$ such that

$$
\left\|\boldsymbol{U}-\boldsymbol{U}_{h}\right\|_{W} \leq C \mathcal{E}(h) .
$$

Proof. The proof is standard, the only particular point being the weak consistency of the method (see, e.g., [6]).

\subsection{Stability and convergence in natural norms}

The next results search prove stability and convergence in a natural norm, in other words, in the norm of the space of the continuous problem, which is not a mesh dependent norm. Since this natural norm does not include any control on the convective terms, stability and convergence in this norm is only meaningful in the case of small cell Reynolds numbers and Weissenberg numbers. In the following, and contrary to what we have been considering up to this point, generic constants $C$ may depend on these numbers and explode as they increase.

Theorem 4.5 (Stability and convergence in natural norms). Suppose that the assumptions of Theorem 4.1 hold and the cell Reynolds numbers and cell Weissenberg numbers are small. Then, the solution of the discrete problem $\boldsymbol{U}_{h}=\left[\boldsymbol{u}_{h}, p_{h}, \boldsymbol{\psi}_{h}\right] \in \mathcal{X}_{h}$ can be bounded as

$$
\sqrt{\eta_{0}}\left\|\boldsymbol{u}_{h}\right\|_{H^{1}(\Omega)}+\frac{\sqrt{\eta_{0}}}{\lambda_{0}}\left\|P_{\psi}\left[\boldsymbol{E} \cdot \boldsymbol{\psi}_{h}\right]\right\|+\frac{1}{\sqrt{\eta_{0}}}\left\|p_{h}\right\| \leq C\left(\frac{1}{\sqrt{\eta_{0}}}\left\|\boldsymbol{f}_{u}\right\|_{H^{-1}(\Omega)}+\frac{\lambda_{0}}{\sqrt{\eta_{0}}}\left\|\boldsymbol{f}_{\psi}\right\|\right) .
$$

Moreover, under the assumptions of Theorem 4.4, if the solution of the continuous problem $\boldsymbol{U}=[\boldsymbol{u}, p, \boldsymbol{\psi}] \in \mathcal{X}$ is regular enough, the following error estimate holds:

$$
\sqrt{\eta_{0}}\left\|\boldsymbol{u}-\boldsymbol{u}_{h}\right\|_{H^{1}(\Omega)}+\frac{\sqrt{\eta_{0}}}{\lambda_{0}}\left\|\boldsymbol{E} \cdot \boldsymbol{\psi}-P_{\psi}\left[\boldsymbol{E} \cdot \boldsymbol{\psi}_{h}\right]\right\|+\frac{1}{\sqrt{\eta_{0}}}\left\|p-p_{h}\right\| \leq C \mathcal{E}(h) .
$$

Proof. Firstly we will prove (4.18). We have that

$$
\begin{aligned}
B_{\text {stab }}\left(\boldsymbol{U}_{h}, \boldsymbol{V}_{h}\right) & =\left\langle\boldsymbol{f}_{u}, \boldsymbol{v}_{h}\right\rangle+\left\langle\boldsymbol{f}_{\psi}, \boldsymbol{\chi}_{h}\right\rangle \\
& \leq C\left(\frac{1}{\sqrt{\eta_{0}}}\left\|\boldsymbol{f}_{u}\right\|_{H^{-1}(\Omega)} \sqrt{\eta_{0}}\left\|\boldsymbol{v}_{h}\right\|_{H^{1}(\Omega)}+\sqrt{\eta_{0}}\left\|\boldsymbol{f}_{\psi}\right\| \frac{\sqrt{\eta_{0}}}{\lambda_{0}}\left\|P_{\psi}\left[\frac{\lambda_{0}}{\eta_{0}} \boldsymbol{E} \cdot \boldsymbol{\chi}_{h}\right]\right\|\right) \\
& \leq C\left(\frac{1}{\sqrt{\eta_{0}}}\left\|\boldsymbol{f}_{u}\right\|_{H^{-1}(\Omega)}+\sqrt{\eta_{0}}\left\|\boldsymbol{f}_{\psi}\right\|\right)\left\|\boldsymbol{V}_{h}\right\|_{W},
\end{aligned}
$$


where $\boldsymbol{V}_{h}=\left[\boldsymbol{v}_{h}, q_{h}, \boldsymbol{\chi}_{h}\right]$ is arbitrary. Therefore, from the inf-sup condition proved in Theorem 4.1 we have

$$
C\left\|\boldsymbol{U}_{h}\right\|_{W}\left\|\boldsymbol{V}_{h}\right\|_{W} \leq B_{\text {stab }}\left(\boldsymbol{U}_{h}, \boldsymbol{V}_{h}\right) \leq C\left(\frac{1}{\sqrt{\eta_{0}}}\left\|\boldsymbol{f}_{u}\right\|_{H^{-1}(\Omega)}+\sqrt{\eta_{0}}\left\|\boldsymbol{f}_{\psi}\right\|\right)\left\|\boldsymbol{V}_{h}\right\|_{W},
$$

and this implies that

$$
\left\|\boldsymbol{U}_{h}\right\|_{W} \leq C\left(\frac{1}{\sqrt{\eta_{0}}}\left\|\boldsymbol{f}_{u}\right\|_{H^{-1}(\Omega)}+\sqrt{\eta_{0}}\left\|\boldsymbol{f}_{\psi}\right\|\right)
$$

Therefore:

$$
\begin{aligned}
\left\|\boldsymbol{U}_{h}\right\|_{W}^{2}= & 2 \beta \eta_{0}\left\|\nabla^{\mathrm{s}} \boldsymbol{u}_{h}\right\|_{K}^{2}+\frac{\eta_{0}(1-\beta)}{\lambda_{0}^{2}}\left\|P_{\psi}\left[\boldsymbol{E} \cdot \boldsymbol{\psi}_{h}\right]\right\|_{K}^{2}+\sum_{K} \alpha_{u}\left\|\rho \boldsymbol{a} \cdot \nabla \boldsymbol{u}_{h}+\nabla p_{h}-\frac{\eta_{p}}{\lambda_{0}} \nabla \cdot P_{\psi}\left[\boldsymbol{E} \cdot \boldsymbol{\psi}_{h}\right]\right\|_{K}^{2} \\
& +\sum_{K} \alpha_{u}\left\|P_{u}^{\perp}\left[\rho \boldsymbol{a} \cdot \nabla \boldsymbol{u}_{h}\right]\right\|_{K}^{2}+\sum_{K} \alpha_{u}\left\|P_{u}^{\perp}\left[\nabla p_{h}\right]\right\|_{K}^{2}+\sum_{K} \alpha_{u}\left\|P_{u}^{\perp}\left[\frac{\eta_{p}}{\lambda_{0}} \nabla \cdot P_{\psi}\left[\boldsymbol{E} \cdot \boldsymbol{\psi}_{h}\right]\right]\right\|_{K}^{2} \\
& +\sum_{K} \alpha_{p}\left\|\nabla \cdot \boldsymbol{u}_{h}\right\|_{K}^{2}+\sum_{K} \alpha_{\psi}\left\|\frac{\lambda}{2 \lambda_{0}}\left(\boldsymbol{a} \cdot \nabla P_{\psi}\left[\boldsymbol{E} \cdot \boldsymbol{\psi}_{h}\right]-\dot{\boldsymbol{\psi}}_{h}^{* *}\right)\right\|_{K}^{2} \\
\leq & C\left(\frac{1}{\sqrt{\eta_{0}}}\left\|\boldsymbol{f}_{u}\right\|_{H^{-1}(\Omega)}+\sqrt{\eta_{0}}\left\|\boldsymbol{f}_{\psi}\right\|\right) .
\end{aligned}
$$

Now, using the inverse inequality, we can write:

$$
\begin{aligned}
\sum_{K} \alpha_{u}\left\|\rho \boldsymbol{a} \cdot \nabla \boldsymbol{u}_{h}+\nabla p_{h}\right\|_{K}^{2} \leq & \sum_{K} \alpha_{u}\left\|\rho \boldsymbol{a} \cdot \nabla \boldsymbol{u}_{h}+\nabla p_{h}-\frac{\eta_{p}}{\lambda_{0}} \nabla \cdot P_{\psi}\left[\boldsymbol{E} \cdot \boldsymbol{\psi}_{h}\right]\right\|_{K}^{2} \\
& +\sum_{K} \alpha_{u} \frac{c_{\mathrm{inv}} \eta_{0}}{\lambda_{0}}\left(\frac{(1-\beta)}{h}\right)^{2} \frac{\eta_{0}}{\lambda_{0}}\left\|P_{\psi}\left[\boldsymbol{E} \cdot \boldsymbol{\psi}_{h}\right]\right\|_{K}^{2} .
\end{aligned}
$$

In this expression we only have control on $\rho \boldsymbol{a} \cdot \nabla \boldsymbol{u}_{h}+\nabla p_{h}$. There is the possibility of bounding the pressure gradient making use of the control over the viscous term, since

$$
\sum_{K} \alpha_{u}\left\|\nabla p_{h}\right\|_{K}^{2} \leq \sum_{K} \alpha_{u}\left\|\rho \boldsymbol{a} \cdot \nabla \boldsymbol{u}_{h}+\nabla p_{h}\right\|_{K}^{2}+\sum_{K} \alpha_{u} \frac{\eta_{0}}{h^{2}}\left(\frac{\rho\|\boldsymbol{a}\|_{L^{\infty}(K)} h}{\eta_{0}}\right)^{2}\left\|\nabla \boldsymbol{u}_{h}\right\|_{K}^{2} .
$$

Note that this expression explodes with the cell Reynolds number $\operatorname{Re}_{K}$. Then, from inequality (4.20), and using Korn's inequality, the expression of $\alpha_{u}$ and taking into account that $0<\beta<1$, we obtain:

$$
\eta_{0}\left\|\boldsymbol{u}_{h}\right\|_{H^{1}(\Omega)}^{2}+\frac{\eta_{0}}{\lambda_{0}^{2}}\left\|P_{\psi}\left[\boldsymbol{E} \cdot \boldsymbol{\psi}_{h}\right]\right\|^{2}+\frac{h^{2}}{\eta_{0}} \sum_{K}\left\|\nabla p_{h}\right\|_{K}^{2} \leq C\left(\frac{1}{\sqrt{\eta_{0}}}\left\|\boldsymbol{f}_{u}\right\|_{H^{-1}(\Omega)}+\sqrt{\eta_{0}}\left\|\boldsymbol{f}_{\psi}\right\|\right)^{2} .
$$

For the $L^{2}$ stability for the pressure we rely on the inf-sup condition between the velocity and pressure spaces that holds for the continuous problem. See the details in [11].

Now we will prove (4.19). Theorem 4.4 implies that $\left\|\boldsymbol{U}-\boldsymbol{U}_{h}\right\|_{W} \leq C \mathcal{E}(h)$, and consequently we have that

$$
\begin{aligned}
\eta_{0}\left\|\boldsymbol{u}-\boldsymbol{u}_{h}\right\|_{H^{1}(\Omega)}^{2} & +\frac{\eta_{0}}{\lambda_{0}^{2}}\left\|\boldsymbol{E} \cdot \boldsymbol{\psi}-P_{\psi}\left[\boldsymbol{E} \cdot \boldsymbol{\psi}_{h}\right]\right\|^{2} \\
& +\sum_{K} \alpha_{u}\left\|\rho \boldsymbol{a} \cdot \nabla\left(\boldsymbol{u}-\boldsymbol{u}_{h}\right)+\nabla\left(p-p_{h}\right)-\frac{\eta_{0}(1-\beta)}{\lambda_{0}} \nabla \cdot\left(\boldsymbol{E} \cdot \boldsymbol{\psi}-P_{\psi}\left[\boldsymbol{E} \cdot \boldsymbol{\psi}_{h}\right]\right)\right\|_{K}^{2} \leq \mathcal{E}^{2}(h) .
\end{aligned}
$$


Now we will follow the same procedure as the used to prove stability; assuming the cell Reynolds number to be small, we get

$$
\begin{aligned}
\sum_{K} \alpha_{u} & \left\|\rho \boldsymbol{a} \cdot \nabla\left(\boldsymbol{u}-\boldsymbol{u}_{h}\right)+\nabla\left(p-p_{h}\right)\right\|_{K}^{2} \\
\leq & \sum_{K} \alpha_{u}\left\|\rho \boldsymbol{a} \cdot \nabla\left(\boldsymbol{u}-\boldsymbol{u}_{h}\right)+\nabla\left(p-p_{h}\right)-\frac{\eta_{0}(1-\beta)}{\lambda_{0}} \nabla \cdot\left(\boldsymbol{E} \cdot \boldsymbol{\psi}-P_{\psi}\left[\boldsymbol{E} \cdot \boldsymbol{\psi}_{h}\right]\right)\right\|_{K}^{2} \\
& +\sum_{K} \alpha_{u} \frac{\eta_{0}}{\lambda_{0}^{2}}\left(\frac{(1-\beta)}{h}\right)^{2} \eta_{0} \varepsilon_{0, K}^{2}(\boldsymbol{\psi}),
\end{aligned}
$$

and following the same reasoning

$$
\sum_{K} \alpha_{u}\left\|\nabla\left(p-p_{h}\right)\right\|_{K}^{2} \leq \sum_{K} \alpha_{u}\left\|\rho \boldsymbol{a} \cdot \nabla\left(\boldsymbol{u}-\boldsymbol{u}_{h}\right)+\nabla\left(p-p_{h}\right)\right\|_{K}^{2}+\sum_{K} \alpha_{u} \frac{\eta_{0}}{h^{2}}\left(\frac{\rho\|\boldsymbol{a}\|_{L^{\infty}(K)} h}{\eta_{0}}\right)^{2} \varepsilon_{1, K}^{2}(\boldsymbol{u}) .
$$

So, we obtain

$$
\eta_{0}\left\|\boldsymbol{u}-\boldsymbol{u}_{h}\right\|_{H^{1}(\Omega)}^{2}+\frac{\eta_{0}}{\lambda_{0}^{2}}\left\|\boldsymbol{E} \cdot \boldsymbol{\psi}-P_{\psi}\left[\boldsymbol{E} \cdot \boldsymbol{\psi}_{h}\right]\right\|^{2}+\frac{h^{2}}{\eta_{0}} \sum_{K}\left\|\nabla\left(p-p_{h}\right)\right\|_{K}^{2} \leq C \mathcal{E}^{2}(h) .
$$

The error estimate to include the $L^{2}$ norm of the pressure error can be obtained following the reasoning indicated in [11].

Theorem 4.6 ( $L^{2}$ error estimate for the velocity). Suppose that the assumptions of Theorem 4.5 hold and the continuous problem satisfies the elliptic regularity condition

$$
\sqrt{\eta_{0}}\|\boldsymbol{u}\|_{H^{2}(\Omega)}+\frac{\sqrt{\eta_{0}}}{\lambda_{0}}\|\boldsymbol{E} \cdot \boldsymbol{\psi}\|_{H^{1}(\Omega)}+\frac{1}{\sqrt{\eta_{0}}}\|p\|_{H^{1}(\Omega)} \leq C \frac{1}{\sqrt{\eta_{0}}}\left\|\boldsymbol{f}_{u}\right\| .
$$

Then

$$
\sqrt{\eta_{0}}\left\|\boldsymbol{u}-\boldsymbol{u}_{h}\right\| \leq C h\left(\sqrt{\eta_{0}}\left\|\boldsymbol{u}-\boldsymbol{u}_{h}\right\|_{H^{1}(\Omega)}+\frac{\sqrt{\eta_{0}}}{\lambda_{0}}\left\|\boldsymbol{E} \cdot \boldsymbol{\psi}-P_{\psi}\left[\boldsymbol{E} \cdot \boldsymbol{\psi}_{h}\right]\right\|+\frac{1}{\sqrt{\eta_{0}}}\left\|p-p_{h}\right\|\right) .
$$

Proof. Let $[\boldsymbol{\omega}, \pi, \boldsymbol{S}] \in \mathcal{X}$ be the solution of the following adjoint problem:

$$
\begin{aligned}
\nabla \cdot \boldsymbol{S}-2 \beta \eta_{0} \Delta \boldsymbol{\omega}-\rho \boldsymbol{a} \cdot \nabla \boldsymbol{\omega}-\nabla \pi & =\frac{\eta_{0}}{\ell^{2}}\left(\boldsymbol{u}-\boldsymbol{u}_{h}\right), \\
-\nabla \cdot \boldsymbol{\omega} & =0, \\
\frac{1}{2 \eta_{0}(1-\beta)} \boldsymbol{S}+\nabla^{\mathrm{s}} \boldsymbol{\omega}-\frac{\lambda}{2 \eta_{0}(1-\beta)}\left(\boldsymbol{a} \cdot \nabla \boldsymbol{S}+\boldsymbol{S} \cdot(\nabla \boldsymbol{a})^{T}+\nabla \boldsymbol{a} \cdot \boldsymbol{S}\right) & =\mathbf{0},
\end{aligned}
$$

with $\boldsymbol{\omega}=\mathbf{0}$ on $\partial \Omega$ and where $\ell$ is a characteristic length scale of the problem that has only been introduced to keep the dimensionality. Let also $\left[\tilde{\boldsymbol{\omega}}_{h}, \tilde{\pi}_{h}, \tilde{\boldsymbol{S}}_{h}\right]$ be the best approximation to $[\boldsymbol{\omega}, \pi, \boldsymbol{S}]$ in $\boldsymbol{\mathcal { X }}_{h}$. Testing (4.22) with $\boldsymbol{u}-\boldsymbol{u}_{h},(4.23)$ with $p-p_{h}$ and (4.24) with $\boldsymbol{\psi}-\boldsymbol{\psi}_{h}$, we can obtain the next expression:

$$
\begin{aligned}
\frac{\eta_{0}}{\ell^{2}}\left\|\boldsymbol{u}-\boldsymbol{u}_{h}\right\|^{2}= & B_{\psi}\left(\left[\boldsymbol{u}-\boldsymbol{u}_{h}, p-p_{h}, \boldsymbol{\psi}-\boldsymbol{\psi}_{h}\right],[\boldsymbol{\omega}, \pi, \boldsymbol{S}]\right) \\
= & B_{\mathrm{stab}}\left(\left[\boldsymbol{u}-\boldsymbol{u}_{h}, p-p_{h}, \boldsymbol{\psi}-\boldsymbol{\psi}_{h}\right],[\boldsymbol{\omega}, \pi, \boldsymbol{S}]\right) \\
& -\sum_{K} \alpha_{u}\left\langle P_{u}^{\perp}\left[-\frac{\eta_{p}}{\lambda_{0}} \nabla \cdot P_{\psi}\left[\boldsymbol{E} \cdot \boldsymbol{\psi}-\boldsymbol{E} \cdot \boldsymbol{\psi}_{h}\right]\right], P_{u}^{\perp}[-\nabla \cdot \boldsymbol{S}]\right\rangle_{K}
\end{aligned}
$$




$$
\begin{aligned}
& -\sum_{K} \alpha_{u}\left\langle P_{u}^{\perp}\left[\nabla\left(p-p_{h}\right)\right], P_{u}^{\perp}[\nabla \pi]\right\rangle_{K}-\sum_{K}\left\langle P_{u}^{\perp}\left[\rho \boldsymbol{a} \cdot \nabla\left(\boldsymbol{u}-\boldsymbol{u}_{h}\right)\right], P_{u}^{\perp}[\rho \boldsymbol{a} \cdot \nabla \boldsymbol{\omega}]\right\rangle_{K} \\
& -\sum_{K} \alpha_{p}\left\langle P_{p}^{\perp}\left[\nabla \cdot\left(\boldsymbol{u}-\boldsymbol{u}_{h}\right)\right], P_{p}^{\perp}[\nabla \cdot \boldsymbol{\omega}]\right\rangle_{K} \\
& -\sum_{K} \alpha_{\psi}\left\langle-\nabla^{\mathrm{s}}\left(\boldsymbol{u}-\boldsymbol{u}_{h}\right)+\frac{\lambda}{2 \lambda_{0}}\left(\boldsymbol{a} \cdot \nabla P_{\psi}\left[\boldsymbol{E} \cdot \boldsymbol{\psi}-\boldsymbol{E} \cdot \boldsymbol{\psi}_{h}\right]-P_{\psi}\left[\boldsymbol{E} \cdot \boldsymbol{\psi}-\boldsymbol{E} \cdot \boldsymbol{\psi}_{h}\right] \cdot \nabla \boldsymbol{a}\right.\right. \\
& \left.\left.-(\nabla \boldsymbol{a})^{T} \cdot P_{\psi}\left[\boldsymbol{E} \cdot \boldsymbol{\psi}-\boldsymbol{E} \cdot \boldsymbol{\psi}_{h}\right]\right),-\nabla^{\mathrm{s}} \boldsymbol{\omega}+\frac{\lambda}{2 \eta_{p}}\left(\boldsymbol{a} \cdot \nabla \boldsymbol{S}+\boldsymbol{S} \cdot(\nabla \boldsymbol{a})^{T}+\nabla \boldsymbol{a} \cdot \boldsymbol{S}\right)\right\rangle_{K},
\end{aligned}
$$

where we have used the definition of $B_{\text {stab }}$ in (3.7). The fifth and sixth terms of (4.25) are zero because of (4.23) and (4.24), respectively. Therefore only four terms need to be bounded. Considering (3.9), these can be written as follows

$$
\begin{aligned}
\frac{\eta_{0}}{\ell^{2}}\left\|\boldsymbol{u}-\boldsymbol{u}_{h}\right\|^{2}= & B_{\text {stab }}\left(\left[\boldsymbol{u}-\boldsymbol{u}_{h}, p-p_{h}, \boldsymbol{\psi}-\boldsymbol{\psi}_{h}\right],[\boldsymbol{\omega}, \pi, \boldsymbol{S}]\right) \\
& -\sum_{K} \alpha_{u}\left\langle P_{u}^{\perp}\left[-\frac{\eta_{p}}{\lambda_{0}} \nabla \cdot P_{\psi}\left[\boldsymbol{E} \cdot \boldsymbol{\psi}-\boldsymbol{E} \cdot \boldsymbol{\psi}_{h}\right]\right], P_{u}^{\perp}[-\nabla \cdot \boldsymbol{S}]\right\rangle_{K} \\
& -\sum_{K} \alpha_{u}\left\langle P_{u}^{\perp}\left[\nabla\left(p-p_{h}\right)\right], P_{u}^{\perp}[\nabla \pi]\right\rangle_{K}-\sum_{K}\left\langle P_{u}^{\perp}\left[\rho \boldsymbol{a} \cdot \nabla\left(\boldsymbol{u}-\boldsymbol{u}_{h}\right)\right], P_{u}^{\perp}[\rho \boldsymbol{a} \cdot \nabla \boldsymbol{\omega}]\right\rangle_{K} .
\end{aligned}
$$

Using the interpolation properties and the shift assumption (4.21) it follows that

$$
\begin{aligned}
\left\|\boldsymbol{\omega}-\tilde{\boldsymbol{\omega}}_{h}\right\|_{H^{1}(\Omega)} & \leq C h\|\boldsymbol{\omega}\|_{H^{2}(\Omega)} \leq C h \frac{1}{\ell^{2}}\left\|\boldsymbol{u}-\boldsymbol{u}_{h}\right\|, \\
\left\|\boldsymbol{S}-\tilde{\boldsymbol{S}}_{h}\right\| & \leq C h\|\boldsymbol{S}\|_{H^{1}(\Omega)} \leq C h \frac{\eta_{0}}{\ell^{2}}\left\|\boldsymbol{u}-\boldsymbol{u}_{h}\right\|, \\
\left\|\pi-\tilde{\pi}_{h}\right\| & \leq C h\|\pi\|_{H^{1}(\Omega)} \leq C h \frac{\eta_{0}}{\ell^{2}}\left\|\boldsymbol{u}-\boldsymbol{u}_{h}\right\| .
\end{aligned}
$$

From these expressions we obtain

$$
\begin{aligned}
& B_{\text {stab }}\left(\left[\boldsymbol{u}-\boldsymbol{u}_{h}, p-p_{h}, \boldsymbol{\psi}-\boldsymbol{\psi}_{h}\right],[\boldsymbol{\omega}, \pi, \boldsymbol{S}]\right) \\
&=B_{\text {stab }}\left(\left[\boldsymbol{u}-\boldsymbol{u}_{h}, p-p_{h}, \boldsymbol{\psi}-\boldsymbol{\psi}_{h}\right],\left[\boldsymbol{\omega}-\tilde{\boldsymbol{\omega}}_{h}, \pi-\tilde{\pi}_{h}, \boldsymbol{S}-\tilde{\boldsymbol{S}}_{h}\right]\right) \\
&-\underbrace{\sum_{K} \alpha_{u}\left\langle P_{u}^{\perp}\left[-\frac{\eta_{p}}{\lambda_{0}} \nabla \cdot P_{\psi}\left[\boldsymbol{E} \cdot \boldsymbol{\psi}-\boldsymbol{E} \cdot \boldsymbol{\psi}_{h}\right]\right], P_{u}^{\perp}\left[-\nabla \cdot\left(\boldsymbol{S}-\tilde{\boldsymbol{S}}_{h}\right)\right]\right\rangle_{K}}_{(2)} \\
&-\underbrace{\sum_{K} \alpha_{u}\left\langle P_{u}^{\perp}\left[\nabla\left(p-p_{h}\right)\right], P_{u}^{\perp}\left[\nabla\left(\pi-\tilde{\pi}_{h}\right)\right]\right\rangle_{K}}_{(3)}-\underbrace{\sum_{K}\left\langle P_{u}^{\perp}\left[\boldsymbol{a} \cdot \nabla\left(\boldsymbol{u}-\boldsymbol{u}_{h}\right)\right], P_{u}^{\perp}\left[\boldsymbol{a} \cdot \nabla\left(\boldsymbol{\omega}-\tilde{\boldsymbol{\omega}}_{h}\right)\right]\right\rangle_{K}}_{(4)} .
\end{aligned}
$$

Considering these inequalities we can bound terms (2)-(4) easily:

$$
\begin{aligned}
(2) & \leq \sum_{K} \alpha_{u} \frac{\eta_{p}}{\lambda_{0}} \frac{1}{h}\left\|P_{\psi}\left[\boldsymbol{E} \cdot \boldsymbol{\psi}-\boldsymbol{E} \cdot \boldsymbol{\psi}_{h}\right]\right\|_{K} \frac{\eta_{0}}{\ell^{2}}\left\|\boldsymbol{u}-\boldsymbol{u}_{h}\right\|_{K} \\
& \leq \sum_{K} \alpha_{u} \frac{\eta_{p}}{\lambda_{0}} \frac{1}{h}\left\|\boldsymbol{E} \cdot \boldsymbol{\psi}-P_{\psi}\left[\boldsymbol{E} \cdot \boldsymbol{\psi}_{h}\right]\right\|_{K} \frac{\eta_{0}}{\ell^{2}}\left\|\boldsymbol{u}-\boldsymbol{u}_{h}\right\|_{K}, \\
(3) & \leq \sum_{K} \alpha_{u} \frac{1}{h}\left\|p-p_{h}\right\|_{K} \frac{\eta_{0}}{\ell^{2}}\left\|\boldsymbol{u}-\boldsymbol{u}_{h}\right\|_{K},
\end{aligned}
$$




$$
(4) \leq \sum_{K} \alpha_{u}\left(\rho\|\boldsymbol{a}\|_{L^{\infty}(K)}\right)^{2} \frac{h}{\ell^{2}}\left\|\boldsymbol{u}-\boldsymbol{u}_{h}\right\|_{K}\left\|\boldsymbol{u}-\boldsymbol{u}_{h}\right\|_{H^{1}(\Omega)} .
$$

We have to bound the terms of

$$
B_{\text {stab }}\left(\left[\boldsymbol{u}-\boldsymbol{u}_{h}, p-p_{h}, \boldsymbol{\psi}-\boldsymbol{\psi}_{h}\right],\left[\boldsymbol{\omega}-\tilde{\boldsymbol{\omega}}_{h}, \pi-\tilde{\pi}_{h}, \boldsymbol{S}-\tilde{\boldsymbol{S}}_{h}\right]\right),
$$

for which similar techniques to those used before. Finally, combining the bounds obtained in (4.26) yields:

$$
\begin{aligned}
\frac{\eta_{0}}{l^{2}}\left\|\boldsymbol{u}-\boldsymbol{u}_{h}\right\|^{2} \leq & h \frac{\sqrt{\eta_{0}}}{\ell^{2}}\left\|\boldsymbol{u}-\boldsymbol{u}_{h}\right\|\left(\sqrt{\eta_{0}}\left\|\boldsymbol{u}-\boldsymbol{u}_{h}\right\|_{H^{1}(\Omega)}+\sum_{K}\left(\frac{\rho\|\boldsymbol{a}\|_{L^{\infty}(K)}}{\eta_{0}}\right) \sqrt{\eta_{0}}\left\|\boldsymbol{u}-\boldsymbol{u}_{h}\right\|_{H^{1}(K)}\right. \\
& +\frac{\sqrt{\eta_{0}}}{\lambda_{0}}\left\|\boldsymbol{E} \cdot \boldsymbol{\psi}-P_{\psi}\left[\boldsymbol{E} \cdot \boldsymbol{\psi}_{h}\right]\right\|_{K}+\frac{\sqrt{\eta_{0}}}{\lambda_{0}} \sum_{K}\left(\frac{\lambda\|\boldsymbol{a}\|_{L^{\infty}(K)}}{h}\right)\left\|\boldsymbol{E} \cdot \boldsymbol{\psi}-P_{\psi}\left[\boldsymbol{E} \cdot \boldsymbol{\psi}_{h}\right]\right\|_{K} \\
& \left.+\frac{1}{\sqrt{\eta_{0}}}\left\|p-p_{h}\right\|_{K}\right),
\end{aligned}
$$

and theorem follows. Note again that the bound obtained explodes with the cell Reynolds and the cell Weissenberg numbers.

\section{Conclusions}

In this paper we have analyzed the finite element formulation proposed in [28] applied to a linearized form of the logarithmic reformulation of the viscoelastic flow problem. A similar analysis was done in [6] for the standard formulation, and thus the present paper can be considered a follow-up of the latter. Despite the linearization and the various assumptions that have been needed in our analysis, it serves to draw two main conclusions. The first is that the finite element formulation proposed is effective as stabilization technique, as it allows one to use arbitrary interpolations for all variables in play (we have considered for simplicity the case of continuous interpolations) and yields optimal error estimates, both in the stabilized norm and in the natural norm of the problem, in the spaces in which the continuous problem is posed. The second conclusion is that the logarithmic reformulation has a significantly better behavior in terms of the Weissenberg number than the standard one, with an error estimate that deteriorates much more slowly when this number increases; this provides some theoretical foundation to justify the use of the logarithmic reformulation to attempt the HWNP, at least using the FE formulation we have introduced.

Acknowledgements. R. Codina gratefully acknowledges the support received from the ICREA Acadèmia Program, from the Catalan Government. L. Moreno acknowledges the support received from the Spanish Government through a predoctoral FPI Grant.

\section{REFERENCES}

[1] J. Baranger and D. Sandri, Finite element approximation of viscoelastic fluid flow: existence of approximate solutions and error bounds. Numer. Math. 63 (1992) 13-27.

[2] G. Barrenechea, E. Castillo and R. Codina, Time-dependent semi-discrete analysis of the viscoelastic fluid flow problem using a variational multiscale stabilized formulation. IMA J. Numer. Anal. 39 (2019) 792-819.

[3] M.A. Behr, L.P. Franca and T.E. Tezduyar, Stabilized finite element methods for the velocity-pressure-stress formulation of incompressible flows. Comput. Methods Appl. Mech. Eng. 104 (1993) 31-48.

[4] F. Brezzi and M. Fortin, Mixed and Hybrid Finite Element Methods. Springer Science \& Business Media (1991).

[5] E. Castillo and R. Codina, Variational multi-scale stabilized formulations for the stationary three-field incompressible viscoelastic flow problem. Comput. Methods Appl. Mech. Eng. 279 (2014) 579-605.

[6] E. Castillo and R. Codina, Numerical analysis of a stabilized finite element approximation for the three-field linearized viscoelastic fluid problem using arbitrary interpolations. ESAIM: M2AN 51 (2017) 1407-1427. 
[7] E. Castillo and R. Codina, Finite element approximation of the viscoelastic flow problem: a non-residual based stabilized formulation. Comput. Fluids 142 (2017) 72-78.

[8] R. Codina, Stabilization of incompressibility and convection through orthogonal sub-scales in finite element methods. Comput. Methods Appl. Mech. Eng. 190 (2000) 1579-1599.

[9] R. Codina, Stabilized finite element approximation of transient incompressible flows using orthogonal subscales. Comput. Methods Appl. Mech. Eng. 191 (2002) 4295-4321.

[10] R. Codina, Analysis of a stabilized finite element approximation of the Oseen equations using orthogonal subscales. Appl. Numer. Math. 58 (2008) 264-283.

[11] R. Codina, Finite element approximation of the three-field formulation of the Stokes problem using arbitrary interpolations. SIAM J. Numer. Anal. 47 (2009) 699-718.

[12] R. Codina, S. Badia, J. Baiges and J. Principe, Variational multiscale methods in computational fluid dynamics, edited by E. Stein, R. Borst and T.J.R. Hughes. In: Encyclopedia of Computational Mechanics. John Wiley \& Sons Ltd. (2017) 1-28.

[13] V.J. Ervin and W.W. Miles, Approximation of time-dependent viscoelastic fluid flow: SUPG approximation. SIAM J. Numer. Anal. 41 (2003) 457-486.

[14] R. Fattal and R. Kupferman, Constitutive laws for the matrix-logarithm of the conformation tensor. J. Non-Newtonian Fluid Mech. 123 (2004) 281-285.

[15] R. Fattal and R. Kupferman, Time-dependent simulation of viscoelastic flows at high weissenberg number using the logconformation representation. J. Non-Newtonian Fluid Mech. 126 (2005) 23-37.

[16] E. Fernández-Cara, F. Guillén and R.R. Ortega, Mathematical modeling and analysis of viscoelastic fluids of the Oldroyd kind (2002).

[17] M. Fortin and R. Pierre, On the convergence of the mixed method of crochet and marchal for viscoelastic flows. Comput. Methods Appl. Mech. Eng. 73 (1989) 341-350.

[18] C. Guillopé and J.C. Saut, Global existence and one-dimensional nonlinear stability of shearing motions of viscoelastic fluids of Oldroyd type. ESAIM: M2AN 24 (1990) 369-401.

[19] M. Hieber, Y. Naito and Y. Shibata, Global existence results for Oldroyd-B fluids in exterior domains. J. Differ. Equ. 252 (2012) 2617-2629.

[20] T.J.R. Hughes, G.R. Feijóo, L. Mazzei and J. Quincy, The variational multiscale method. A paradigm for computational mechanics. Comput. Methods Appl. Mech. Eng. 166 (1998) 3-24.

[21] M.A. Hulsen, A.P.G. Van Heel and B.H.A.A. Van Den Brule, Simulation of viscoelastic flows using Brownian configuration fields. J. Non-Newtonian Fluid Mech. 70 (1997) 79-101.

[22] J. Kwack and A. Masud, A three-field formulation for incompressible viscoelastic fluids. Int. J. Eng. Sci. 48 (2010) $1413-1432$.

[23] J. Kwack, A. Masud and K.R. Rajagopal, Stabilized mixed three-field formulation for a generalized incompressible Oldroyd-B model. Int. J. Numer. Methods Fluids 83 (2017) 704-734.

[24] Y. Kwon, Recent results on the analysis of viscoelastic constitutive equations. Korea-Aust. Rheol. J. 14 (2002) $33-45$.

[25] A.I. Leonov, Analysis of simple constitutive equations for viscoelastic liquids. J. Non-Newtonian Fluid Mech. 42 (1992) $323-350$.

[26] M. Lukáčová-Medvidová, H. Mizerová, B. She and J. Stebel, Error analysis of finite element and finite volume methods for some viscoelastic fluids. J. Numer. Math. 24 (2016) 105-123.

[27] J.M. Marchal and M.J. Crochet, A new mixed finite element for calculating viscoelastic flow. J. Non-Newtonian Fluid Mech. 26 (1987) $77-114$.

[28] L. Moreno, R. Codina, J. Baiges and E. Castillo, Logarithmic conformation reformulation in viscoelastic flow problems approximated by a VMS-type stabilized finite element formulation. Comput. Methods Appl. Mech. Eng. 354 (2019) $706-731$.

[29] R.G. Owens and T.N. Phillips, Computational Rheology. World Scientific 14 (2002).

[30] M. Picasso and J. Rappaz, Existence, a priori and a posteriori error estimates for a nonlinear three-field problem arising from Oldroyd-B viscoelastic flows. ESAIM: M2AN 35 (2001) 879-897.

[31] M. Renardy, Mathematical analysis of viscoelastic flows. Annu. Rev. Fluid Mech. 21 (1989) 21-34.

[32] V. Ruas, Une méthode mixte contrainte-déplacement-pression pour la résolution de problemes de viscoélasticité incompressible en déformations planes. C. R. Acad. Sci. Sér. 2: Méc. Phys. Chim. Sci. Univ. Sci. Terre 301 (1985) 1171-1174.

[33] P. Saramito, On a modified non-singular log-conformation formulation for Johnson-Segalman viscoelastic fluids. J. NonNewtonian Fluid Mech. 211 (2014) 16-30. 